\title{
A Computer Science Perspective on Digital Transformation in Production
}

\author{
PHILIPP BRAUNER and MANUELA DALIBOR, RWTH Aachen University, Germany \\ MATTHIAS JARKE, RWTH Aachen University, Germany and Fraunhofer FIT, Germany \\ IKE KUNZE, ISTVÁN KOREN, GERHARD LAKEMEYER, MARTIN LIEBENBERG, \\ JUDITH MICHAEL, and JAN PENNEKAMP, RWTH Aachen University, Germany \\ CHRISTOPH QUIX, Fraunhofer FIT, Germany and Hochschule Niederrhein, Germany \\ BERNHARD RUMPE, RWTH Aachen University, Germany \\ WIL VAN DER AALST, RWTH Aachen University, Germany and Fraunhofer FIT, Germany \\ KLAUS WEHRLE, RWTH Aachen University, Germany \\ ANDREAS WORTMANN, RWTH Aachen University, Germany and University of Stuttgart, Germany \\ MARTINA ZIEFLE, RWTH Aachen University, Germany
}

\begin{abstract}
The Industrial Internet-of-Things (IIoT) promises significant improvements for the manufacturing industry by facilitating the integration of manufacturing systems by Digital Twins. However, ecological and economic demands also require a cross-domain linkage of multiple scientific perspectives from material sciences, engineering, operations, business, and ergonomics, as optimization opportunities can be derived from any of these perspectives. To extend the IIoT to a true Internet of Production, two concepts are required: first, a complex, interrelated network of Digital Shadows which combine domain-specific models with data-driven AI methods; and second, the integration of a large number of research labs, engineering, and production sites as a World Wide Lab which offers controlled exchange of selected, innovation-relevant data even across company boundaries. In this article, we define the underlying Computer Science challenges implied by these novel concepts in four layers: Smart human interfaces provide access to information that has been generated by model-integrated AI. Given the large variety of manufacturing data, new data modeling techniques should enable efficient management of Digital Shadows, which is supported by an interconnected infrastructure. Based on a detailed analysis of these challenges, we derive a systematized research roadmap to make the vision of the Internet of Production a reality.
\end{abstract}

Funded by the Deutsche Forschungsgemeinschaft (DFG, German Research Foundation) under Germany's Excellence Strategy - EXC-2023 Internet of Production - 390621612.

Authors' addresses: P. Brauner, M. Dalibor, I. Kunze, I. Koren (corresponding author), G. Lakemeyer, M. Liebenberg, J. Michael, J. Pennekamp, B. Rumpe, K. Wehrle, and M. Ziefle, RWTH Aachen University, 52062 Aachen, Germany; emails: brauner@comm.rwth-aachen.de, dalibor@se-rwth.de, ike.kunze@comsys.rwth-aachen.de, koren@pads.rwth-aachen.de, gerhard@cs.rwth-aachen.de, liebenberg@kbsg.rwth-aachen.de, michael@se-rwth.de, jan.pennekamp@comsys.rwthaachen.de, rumpe@se-rwth.de, \{klaus.wehrle\}@comsys.rwth-aachen.de, ziefle@comm.rwth-aachen.de; M. Jarke and W. van der Aalst, RWTH Aachen University, Germany and Fraunhofer FIT, Germany; emails: jarke@dbis.rwthaachen.de, vdaalst@pads.rwth-aachen.de; C. Quix, Fraunhofer FIT, 53757 Sankt Augustin, Germany; email: christoph.quix@fit.fraunhofer.de; A. Wortmann, RWTH Aachen University, 52062 Aachen, Germany, University of Stuttgart, 70174 Stuttgart, Germany; email: wortmann@se-rwth.de.

Permission to make digital or hard copies of all or part of this work for personal or classroom use is granted without fee provided that copies are not made or distributed for profit or commercial advantage and that copies bear this notice and the full citation on the first page. Copyrights for components of this work owned by others than the author(s) must be honored. Abstracting with credit is permitted. To copy otherwise, or republish, to post on servers or to redistribute to lists, requires prior specific permission and/or a fee. Request permissions from permissions@acm.org.

(c) 2022 Copyright held by the owner/author(s). Publication rights licensed to ACM.

2577-6207/2022/02-ART15 \$15.00

https://doi.org/10.1145/3502265 
CCS Concepts: • Applied computing $\rightarrow$ Engineering; • Computing methodologies $\rightarrow$ Artificial intelligence; • Human-centered computing $\rightarrow$ Human computer interaction (HCI); ・ Information systems $\rightarrow$ Data management systems; • Networks $\rightarrow$ World Wide Web (network structure); • Social and professional topics $\rightarrow$ Socio-technical systems; • Software and its engineering $\rightarrow$ Software system structures;

Additional Key Words and Phrases: Internet of production, world wide lab, digital shadows, industrial internet of things

ACM Reference format:

Philipp Brauner, Manuela Dalibor, Matthias Jarke, Ike Kunze, István Koren, Gerhard Lakemeyer, Martin Liebenberg, Judith Michael, Jan Pennekamp, Christoph Quix, Bernhard Rumpe, Wil van der Aalst, Klaus Wehrle, Andreas Wortmann, and Martina Ziefle. 2022. A Computer Science Perspective on Digital Transformation in Production. ACM Trans. Internet Things 3, 2, Article 15 (February 2022), 32 pages.

https://doi.org/10.1145/3502265

\section{INTRODUCTION}

Motivation and Relevance. Industry 4.0 is considered as the fourth industrial revolution focusing on integrating cyber-physical production systems (CPPS) with processes and stakeholders across the complete value-added chain. The term was announced in 2011 as part of the high-tech strategy of the German Federal Ministry for Education and Research [26] and became an international phenomenon reflected in the Japanese Industrial Value Chain Initiative [71], the Advanced Manufacturing Initiative in the USA [108], the Made in China 2025 strategy [101], the South Korean Manufacturing 3.0 [72], and the UK Catapult research center on High Value Manufacturing [30].

A central challenge in Industry 4.0 is aggregating, abstracting, and analyzing the heterogeneous data required to understand and optimize the processes at hand [35, 57, 140]. However, the required data is often locked up in silos owned by different interdisciplinary stakeholders. Apart from the isolation of data within silos, utilizing information is traditionally limited to specific phases of the product's lifecycle, i.e., development, production, and usage. Hence, the information carried by these data is difficult to identify, interpret, and integrate, which prevents, for instance, using it for cross-functional analytics, human-in-the-loop decision-making, linkage of data with heterogeneous semantics and structures, machine learning applications, or simple integrated visualization to improve production processes. The semantic integration of this information is crucial to provide a comprehensive picture to decision-makers across the value-added chain.

In this article, we introduce the unprecedented concept, challenges, and approaches of designing the Inte Rne t of Production (IoP) [121] that builds on the ideas of the Internet and the Internet of Things (IoT) to facilitate transparent interconnectivity of production systems. To extend the Industrial IoT (IIoT) and similar initiatives to a true $I o P$, two concepts are required: firstly, a complex, interrelated network of Digital Shadows, which combine domain-specific models with focused data-driven AI methods inferred by autonomous agents; and second, the integration of a large number of research labs, engineering, and production sites as a World Wide Lab (WWL), which offers controlled exchange of selected, innovation-relevant data even across company and national boundaries. The IoP intends to interconnect all production activities to unlock advances resulting from information exchange and transfer of knowledge across the complete lifecycles of products, processes, and resources. To this end, the IoP provides models and interfaces to reliably integrate, analyze, and use production data and information throughout time and space dimensions. It fosters cross-domain collaboration on multiple levels, across stakeholders, ideally in realtime. As outlined, these crucial challenges of the IoP are not covered by today's predominant IoT approaches. 
Motivating Example. We illustrate the advantages of the IoP with the example of the ongoing shift towards electric vehicles in the automotive industry, which is inherently characterized by multi-causal uncertainties. While this evolving market is highly attractive for automotive manufacturing companies, suppliers, and infrastructure providers, changing regulations, ambiguous customer demands, and a stream of new technical developments require a rapid adaptation of products and production processes [23, 144]. Today's electric car models consist of a multitude of components and materials, such as aluminum alloys and carbon composites, which must be assembled according to the specifications of increasingly individual customer orders. Each of these materials and components is processed differently, requiring a high degree of flexibility in the assembly line, the preceding supply chain, and the managing systems. These challenges have to be addressed in short-term (e.g., machine configuration), medium-term (e.g., response to customer demands), or long-term decisions (e.g., strategy for new model variants). Rapid and frequent changes hereby imply that the traditional differentiation of the product cycle into distinct development, production, and usage phases is hardly possible, as the different phases are now closely intertwined. Therefore, the full benefits of the IoP can only be realized if a data, service, and analysis infrastructure is established that can provide the required information, which is necessary to make the appropriate decisions. For example, necessary adaptations to the clearance of a car door, based on customer feedback, imply changes in machine parameters and in the supply chain if new materials are demanded. Such a change may affect various stakeholders, e.g., designers, quality managers, shop floor workers, factory planners, sales experts, logistics partners, or suppliers.

Contributions. Two essential concepts enable the IoP: The WWL and Digital Shadows. (1) Corresponding to the relationship of the Internet and the World Wide Web (WWW), we envision the WWL as a core element and major application of the IoP. The WWL aims to be a network of multi-site labs in which models and data from experiments, manufacturing, and usage are made accessible even across company borders to gain additional knowledge. This change will increase the productivity in a similar way as the WWW increased the efficiency of e-commerce transactions, customer interactions, supply chain management, and so on. (2) As a main driver of the WWL, we leverage task- and context-dependent, purpose-driven, aggregated, multi-perspective, and persistent datasets which we call Digital Shadows [92]. We postulate that Digital Shadows are a suitable solution for production engineering applications, as multi-modal views with task-specific granularity can provide high performance, low latency, security, and privacy at the same time.

In this article, we discuss the manifold research challenges towards an IoP comprising a WWL built on Digital Shadows from a computer science perspective. Therefore, we augment current research efforts in manufacturing with a dedicated analysis focusing on computer science challenges and potential contributions towards the IoP. Thereby, we provide engineers with novel insights into fundamental challenges that are related to data processing and information exchange that cross-cut the various partial solutions towards integrated production. Likewise, we provide computer scientists with an analysis highlighting future interdisciplinary research directions to successfully turn the ambitious digital transformation of production into reality.

Therefore, we distinguish four perspectives that address how data will be collected, processed, and transmitted efficiently in the WWL:

(1) Human-computer interaction plays a major role as the complex, heterogeneous, and interconnected information carried by data from production planning and operation has to be presented in a meaningful way to decision-makers; their feedback needs to be collected in smart user interfaces, integrated into the Digital Shadow, and used for production control.

(2) Model-integrated Artificial Intelligence (AI) as synergy between data-driven AI methods and model order reduction techniques from engineering must be considered to enrich 
data semantically, to analyze it, to derive new insights, and to act appropriately in production.

(3) Model-driven engineering is a quintessential prerequisite to relate data to knowledge made explicit in heterogeneous models provided by different stakeholders and communicate it to systems engineers, designers, suppliers, and others.

(4) Aspects of network infrastructure, edge computing, and data management have to be addressed to provide an efficient basic infrastructure for data processing within the IoP.

The holistic horizontal and vertical integration in the IoP offers various tangible benefits to all stakeholders in a production network-from companies in a value-added chain to the individual machines and its operators: increased efficiency and closer integration through better exchange of information between different, previously less integrated, stakeholders and thus higher resource utilization, faster adjustments of the production to change, and less capital commitment. The exploitation of data from the development, production, and usage cycle of products facilitate optimization of future products and processes towards lower costs or capital commitment, higher time, material, and energy efficiency, or higher product quality. Furthermore, cross-learning and semantic knowledge about commonalities and differences between different materials, production processes, and products and their interrelationships will contribute to a smarter production. To sum up, our contributions to the IoP from a computer science perspective are:

- The introduction of the concept of Digital Shadows as an enabler of the IoP.

- The introduction of the concept of WWLs to make knowledge globally accessible.

- A discussion of research challenges for the development of the IoP.

- A set of strategic research directions for the IoP.

Article Organization. We present our approach to implement the IoP. In the following, Section 2 introduces the context of the IoP, before Section 3 explains the IoP, and Section 4 introduces Digital Shadows. Afterward, Section 5 details our concept of the WWL, and Section 6 discusses challenges towards it. Based on these insights, Section 7 presents a strategic research roadmap and, finally, Section 8 concludes.

\section{CONTEXT}

The differences between the terms production and manufacturing are not clearly defined in mechanical engineering. Production is understood as "the conversion of inputs into finished products" [60]. In the US perception [79], manufacturing is "a series of interrelated activities and operations involving the design, material selection, planning, production, quality assurance, management, and marketing of discrete consumer and durable goods" [60]. This definition assumes that manufacturing is broader than production. However, production can also be understood as the broader term including additional activities and operations [79] as, e.g., services can be produced but not manufactured. We follow the latter idea and understand production as the wider term.

The IoP provides semantically adequate and context-aware data for members of production companies and related fields whenever and wherever it is needed [121]. This article focuses on technical requirements for realizing the IoP from a computer science research perspective. Of course, the vision of the IoP can only be successfully addressed, if the new concepts and methods in information technology are applied in an integrated research agenda that also includes new technologies for production engineering. We are part of a research cluster with an extraordinary breadth of more than 30 co-located contributing institutes from different disciplines, such as computer science, engineering, material science, economics, and social-sciences, as well as over 50 industrial partners, such as Robert Bosch GmbH, Samsung Electronics Co. Ltd, and Siemens AG 
Corporate Technology (cf. https://iop.rwth-aachen.de/). We have a holistic perspective on tomorrow's production and also address, e.g., new material compositions for additive manufacturing, the economic perspective of platforms for sharing data between different stakeholders [83], and also ethical implications of our work. We aim at applying the principles inherent to the Internet, such as openness, world-wide access, and community-driven standards to the IoP to achieve a sustainable and effective digital transformation.

\subsection{Digital Twins vs. Digital Shadow}

To realize the IoP, we suggest Digital Twins, which digitally represent material $[15,24]$ and immaterial $[85,95]$ objects and processes of the real world. The challenge here is the integration of the different levels of scale (temporal, spatial, etc.) of the numerous underlying processes, yielding large amounts of data, ill-fitted models, and high latencies if data needs to be aggregated and analyzed. There exist various platforms and approaches to realize Digital Twins [8, 77, 94, 106, 143] or to establish the connection between IoT and Digital Twins, e.g., model-driven approaches for interface generation [81] or the H2020 funded IoTwins Innovation Action project [11], which aims to design a reference architecture for distributed and edge-enabled twins and its evaluation in several industrial test beds.

We do not consider a complete Digital Twin to be feasible due to the massive amounts of data that a virtual replica of a product, machine, or production plant would require. Also, the Digital Twins that are used in practice are not complete digital counterparts of physical objects; rather, they are collections of different datasets and models, each representing a particular aspect of the real object. The datasets are collected for a specific purpose, e.g., sensor data for prediction, CAD models for simulation. To model this scenario more exactly, our vision focuses on Digital Shadows, which we consider as task- and context-dependent, purpose-driven, aggregated, and persistent datasets that encompass a complex reality from multiple perspectives in a more compact fashion and with better performance than a fully integrated Digital Twin (cf. Section 4). A Digital Shadow can be compared to a view in database systems: an aggregated subset of the data of the real object, computed by a complex function that might include complex algorithms for data reduction and analysis.

We have already proposed a conceptual model [10] to describe digital shadows and demonstrate it using a concrete example. The conceptual model was established through interdisciplinary research and intensive discussions and was evaluated in various real-world manufacturing scenarios. It is a foundation to manage complexity, automated analyses, and syntheses, and, ultimately, facilitates cross-domain collaboration. For a better understanding on how Digital Shadows could be used within Digital Twins, we refer the reader to a dedicated example [22].

\subsection{Comparison to State-of-the-Art}

In comparison to existing approaches, the IoP provides a holistic, cross-domain, and collaborative perspective on manufacturing processes. Existing approaches can be categorized into the following areas: Concrete technologies such as the classical Internet, cloud manufacturing and IIoT, business demonstrators and digital transformation strategies such as service-oriented manufacturing approaches, digital manufacturing or the Global Lighthouse Network, and politically and fundingdriven approaches such as Industry 4.0 and other national initiatives.

In comparison to the Classical Internet, the IoP offers more functionality to a restricted group of stakeholders. It grants access to world-wide production-focused knowledge bases, provides aggregated and semantically enriched data from production processes, includes intelligent algorithms and functionalities to support question-solving, and enables users to analyze their own data. These functionalities are available to all stakeholders within production processes, such as 
employees of different companies of the production network within all levels of work, e.g., the workforce, the quality assurance team, marketing experts, production planning, suppliers, or logistics partners.

Certainly, comparing the IoP to the Classical Internet is a fitting analogy given that the Internet itself started as a lab of labs, an aspect that the IoP, in turn, picks up with the concept of the WWL. Moreover, today, the Classical Internet serves as an archetype: It revolutionized networking with its novel idea of packet switching. Other approaches followed up on this idea. For example, in the domain of logistics, the concept of the Physical Internet [7, 109] also relies on the Internet analogy, envisioning to establish a physical form of "packet" switching in logistics. In contrast to the Physical Internet with its cargo and product flows, the IoP itself intends to establish a "knowledge" switching for the manufacturing industry by sourcing information and Digital Shadows from various stakeholders and across domains, with the goal of a sustainable and effective digital transformation.

In relationship to Industry 4.0, the IoP can be seen as a concrete initiative to realize aspects of these strategies in cooperation between research and industry. This includes the integration of digitized CPPSs with their processes and stakeholders to optimize the complete value-added chain. However, this idea is also relevant internationally within the US Advanced Manufacturing Initiative [108], the Chinese Made in China 2025 strategy [101], the Japanese Industrial Value Chain Initiative [71], the South Korean Manufacturing 3.0 [72], and the UK national Catapult research center on High Value Manufacturing [30].

The Global Lighthouse Network [151] is an initiative of the World Economic Forum. The initiative was launched because of the global manufacturing industry's lag in adopting Industry 4.0 technologies. It has essentially the same goals as the IoP, i.e., namely a move toward globally networked production. However, it focuses more on the management perspective, whereas the IoP develops the necessary technical foundations to achieve this goal.

Approaches such as Digital Manufacturing [107] as part of the Fourth Industrial Revolution [136] refer to the digital transformation of production processes using smart and agile manufacturing and smart factories together with digital manufacturing technologies such as additive manufacturing (3D printing), laser cutting, and $\mathrm{CNC}$ processes. In contrast, the IoP has no limit regarding specific manufacturing technologies, and integrates the whole value chain.

IoP and IIoT. General efforts, such as the IIoT or Industry 4.0, typically focus on enabling communication only within the same company [36]. In contrast, our vision of the IoP does not merely intend to enable communication between different companies, but also aims to realize a new level of cross-domain collaboration by enabling the exchange of semantically adequate and context-aware data whenever and wherever it is needed [121].

Cloud Manufacturing is "a service-oriented business model to share manufacturing capabilities and resources on a cloud platform" [48], which encapsulates distributed resources into cloud services, and allows for their integrated management [153]. According to Siderska and Jadaan [139], cloud manufacturing focuses on inter-factory integration, whereas Industry 4.0 also considers intra-factory integration. The IoP shares the Industry 4.0 idea of intra-factory and interfactory integration and it does not restrict its technological approach to only one technology such as cloud applications [48], and service-oriented architectures [134]. Cloud manufacturing platforms can be built for small-medium size enterprises or group enterprises [97]. In contrast to that, the IoP is not limited to one enterprise or a group of enterprises.

Service-oriented Manufacturing integrates services and physical products into one product service system and companies involved focus typically on a specific sector [53]. Research in this area focuses on the business perspective and pricing strategies [154], not the technological and computer science perspective. 
In summary, there are already approaches towards the digital transformation of production. They either focus on singular aspects of digital production, take a management perspective without sufficiently resolving the technical challenges, or lack strategies to enable collaboration across company boundaries. As outlined in the next sections, the IoP integrates these different approaches holistically and provides concepts and method to achieve this ambitious goal.

General Challenges that the aforementioned global initiatives are proposing to solve by integrating manufacturing and IT are of societal and political nature. The proposed solutions have partially overlapping issues, but each also has its strategic foci and thus associated challenges, some of which we outline here. Digital Manufacturing, which aims to integrate digital manufacturing methods into production processes, leads to typical issues in human-robot collaboration like unforeseen events that robots cannot handle [107]. Data availability for monitoring and control, as well as its management and networking are further challenges. Cloud Manufacturing leads to two major challenges [139]: First, general integration issues of cloud computing, IoT, and highperformance computing exist. Second, technical issues such as cloud management engines and visualization in cloud environments surface. For service-oriented manufacturing, Gao et al. [53] discuss challenges in the cooperation between businesses and adaptations of business models for outsourcing parts of the value chain as services. They see service-oriented manufacturing as "innovation from the perspectives of business model, industry insight, and technology advantages".

In Section 6, we specifically describe the challenges involved in realizing the IoP. We categorize these into four layers. In the Outlook, we point out challenges that go beyond these layers, such as implications for business models.

\section{VISION OF THE INTERNET OF PRODUCTION (IoP)}

Modern production environments are characterized by highly complex processes and dependencies along the complete production chain [122]. Consequently, optimizing the overall production requires a performant communication and collaboration not only between different factories of the same company, but also across company boundaries, as otherwise, changes made by one supplier could have negative effects on other companies in the production chain [147]. Rolled out to all stages of the production chain, the IoP would, e.g., allow for faster development cycles, as implications of new design changes can be populated along the overall production chain more easily. Beyond, we identify the main potential of the IoP in the WWL, which combines the information of hundreds to thousands of (different) processes into one huge (virtual) setting.

Establishing and Utilizing a World-Wide Knowledge Base. The resulting world-wide knowledge base consequently enables the usage of data-intensive approaches, such as machine learning, to generate purpose-driven Digital Shadows incorporating deep production knowledge for optimizing processes, efficiently developing new products, or predicting their life span. In today's production landscape, such approaches are not feasible due to the scarcity of available data and the large possible parameter space. This problem is even more pronounced in new production sectors, such as the aforementioned electric vehicle industry, as well as for newly founded companies that do not have a large pool of information. A newly founded electric car manufacturer, for example, would not have many benchmarking opportunities yet many options to innovate. Using the WWL, the manufacturer could now link the current state of its production processes to processes running in factories of other companies (competitors, suppliers, and customers) or also experiments at universities [122] to explore new process improvements. Some of these improvements can be implemented automatically, especially since the concepts originating from the broader context of IIoT, Industry 4.0, and the IoP enable wide-spread factory automation and reconfiguration [149]. Yet, uncalled-for automation limits the optimization of production and processes [137], which is why the impact of human workers should not be underestimated. 


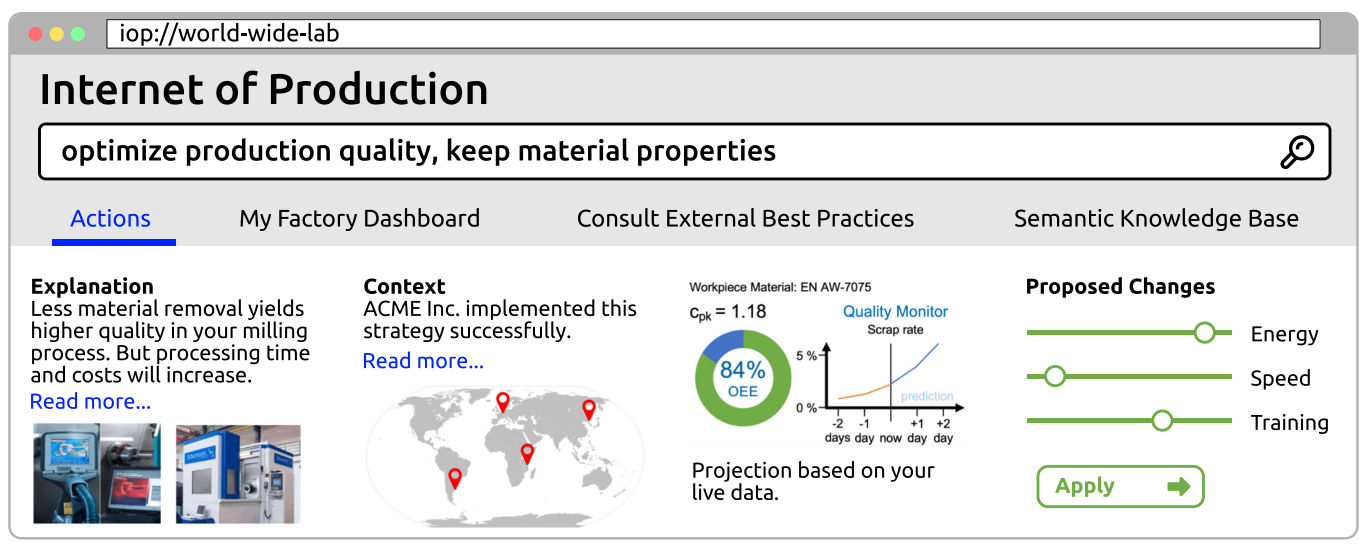

Fig. 1. A mockup of a search engine for production queries leveraging the (global) knowledge base processed by intelligent agents to optimize production processes, as envisioned by the loP.

Access to IoP-Enabled Knowledge. Acknowledging the prominent position of workers and decision-makers in socio-technical systems, we thus put the human at the top of the IoP and do not aim for fully automated production. Consequently, it is important to provide task- and usercentered interfaces to make it easier for human engineers and workers to access the available information and support them in design, manufacturing, and management tasks. One possible interface could be modeled after popular web search engines where users can perform queries to find the desired information. Based on such production queries, data from several, potentially external, sources need to be integrated, semantically enriched, analyzed, and visualized. In the context of the mentioned car manufacturer, operators might ask, e.g., how to optimize production quality while keeping material properties. Figure 1 illustrates our concept using this query. Based on contextual data, e.g., previous queries, the user interface can reference the current material composition and suggest actions. Intelligent software agents [131] behind this interface collect relevant information from the WWL and subsequently analyze the influence of changes in material composition on production as a whole. The data hereby have to be retrieved from various sources maintained by different stakeholders. Access to the data is thus enabled by the WWL. The changes could, e.g., plot a projection of the overall quality of the production. The effects are visually enriched so that the user can understand them more easily. The querying actor could then use the provided information for management decisions regarding changes to the material composition. Proposed changes could be directly applied from within the search user interface.

Establishing the IoP. Smart Human Interfaces are an important component of our envisioned technology stack to enable the IoP, as illustrated on the left side of Figure 2. More specifically, taskand user-centered interfaces are required to facilitate the access of human engineers and workers to the newly gained information. Gathering the corresponding context- and task-specific information itself requires a sophisticated underlying infrastructure. Model-Integrated Artificial Intelligence composes the information in human-understandable form by means of models from different domains combined with AI on the basis of data abstractions and aggregations, which are, in turn, maintained by Data Modeling techniques which themselves need an Interconnected Infrastructure that retrieves and integrates data. Besides the challenges and benefits from an engineering point of view, each of these four layers poses several challenges that are highly relevant from a computer science perspective and that need to be solved on the way to realizing our vision. 


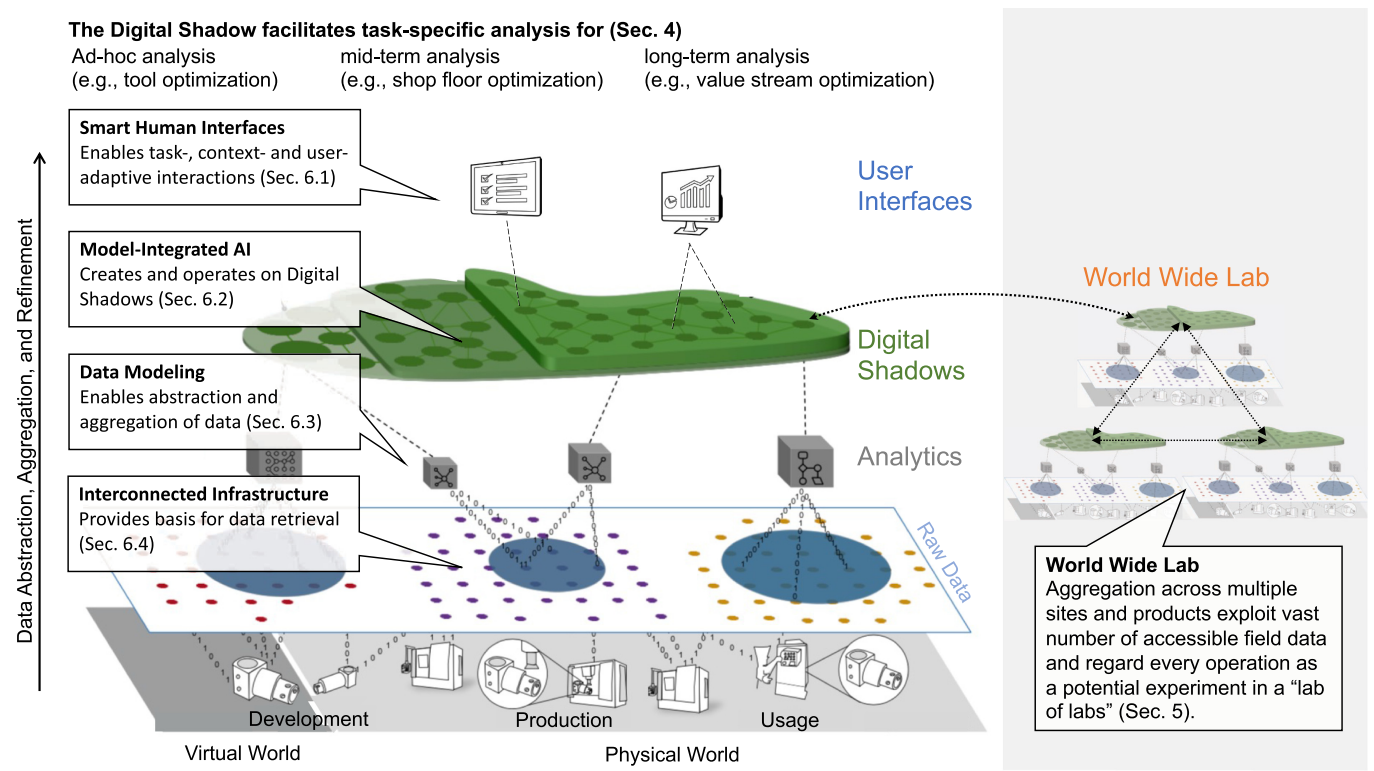

Fig. 2. In the loP, task-adaptive Digital Shadows build on interconnected infrastructure, data modeling, and model-integrated $\mathrm{Al}$ to provide smart interfaces that make the right data actionable at the right time during development, production, and usage. Integration across different production facilities and locations leads to the WWL.

Smart Human Interfaces. As illustrated above, making it easy for human users like production managers and shop floor operators to formulate their problems without the necessity of complicated programming languages requires Smart Human Interfaces. Solutions should be provided in forms that are easy to understand and learn in a micro-learning style [84], so that the human engineer can take appropriate actions to implement them and consequently achieve a real improvement of the production process. What makes this challenging is the volume and diversity of information that needs to be presented: apart from information about material, product, and process optimizations derived from continuous data analysis, we also envision integrating new data from the WWL whenever they are available. Additionally, the current state of the production has to be accounted for, as well as specific input by operators regarding their needs and problems. Thus, the interfaces have to be able to present a diverse set of information to the human workers.

Model-Integrated Al. Based on novel combinations of mathematical models, simulations, and data-driven artificial intelligence, information is gathered from different entities in the WWL, data is aggregated from various sources, Digital Shadows are built [92, 103], and answers to user queries are computed. The implementation of intelligent software agents enables model-integrated AI, meaning various techniques of AI, e.g., knowledge-based systems, machine learning, or data mining, in synergy with engineering models to give answers for specific purposes in production scenarios. The challenges lie in the networking of such agents in the WWL, and the integration of different $\mathrm{AI}$ approaches to provide trustworthy aids for manufacturing.

Data Modeling. Furthermore, sophisticated Data Modeling techniques are required to model the Digital Shadow, i.e., the heterogeneous datasets originating from different production processes. The data models need to be tightly connected to the engineering models of the machines on the 
shop floor, as their setup determines the type and structure of the data to be collected. Thus, in addition to describing the static aspects (e.g., schema, provenance, and quality), the ability to derive the data models from the engineering models by applying model transformations must be considered.

Interconnected Infrastructure. An Interconnected Infrastructure for the WWL is challenged by increasingly high data rates in the manufacturing industry, where sensors can generate data in the range of giga- to peta-bytes per second [55]. These data rates are problematic for storage and semantic analysis in real-time, as well as for sending these vast amounts of data within the WWL. Therefore, data has to be aggregated and reduced in a semantically meaningful way to still enable purpose-driven, meaningfully abstracted and aggregated, temporal data subsets. For this, model and data reduction techniques have to be applied, e.g., in the form of edge computing or in-network processing, to process the data as early as possible in the WWL.

Organizational Challenges. In addition to the requirements of the various layers of the IoP, some general issues also apply to several layers. The stakeholders of the WWL need to establish a level of trust between each other, so that data can be shared [32,52]. Then, a platform with standardized interfaces can be established that provides the technological basis for data exchange. For example, the International Data Spaces (IDS) Association [111, 112] currently develop a platform for secure, trusted, and reliable data exchange while also guaranteeing the data sovereignty of the data providers. We plan to apply some key aspects of this platform in the IoP [73]. When considering the individual local production sites, safety and security aspects play a crucial role as well [63] because a minimum of guarantees must be in place to ensure a smooth and uneventful operation of the WWL.

Summarizing, our vision of the IoP addresses the idea of exchanging data on a global level and using this data to provide task-specific information whenever and wherever needed. Consequently, the form of data representation used in the IoP is a key concept of our vision. In the following, we present how we utilize Digital Shadows to realize our envisioned cross-domain collaboration.

\section{DIGITAL SHADOWS ENABLE THE INTERNET OF PRODUCTION}

Our vision of the IoP demands that the right information is available at the right time, depending on the task and context. Such information includes data from the production systems and processes, shop floor workers, customers, suppliers, and many other sources, which allow optimizing production, reduce downtimes, and save resources [152]. Figure 2 shows the stepwise construction, refinement, and application of Digital Shadows. At the bottom layer, physical and virtual production steps produce raw data that characterize the product, the process, and the resources. Due to the volume, variety, and velocity of data, retrieving the right information from the data is figuratively like searching for a needle in a haystack. Hence, these data need to be abstracted and aggregated to support meaningful decision-making at different levels and scopes, from real-time machine and process optimizations to long-term strategic planning. An interconnected infrastructure, including additional metadata characterizing data points and facilitating remote access, builds the basis for data aggregation. Data models provide structural information about the available data and thus enable knowledge gain via purposeful connection of data points. By applying AI methods, such as machine learning or process mining [145], we can attain further knowledge from the available data, e.g., quality predictions or bottlenecks in assembly lines. Human interfaces support decision making, process optimization, error avoidance, and thus improve production performance by providing user- and target-specific Digital Shadows. They also facilitate human interaction with the CPPS and allow for analytics and AI methods creating Digital Shadows. 


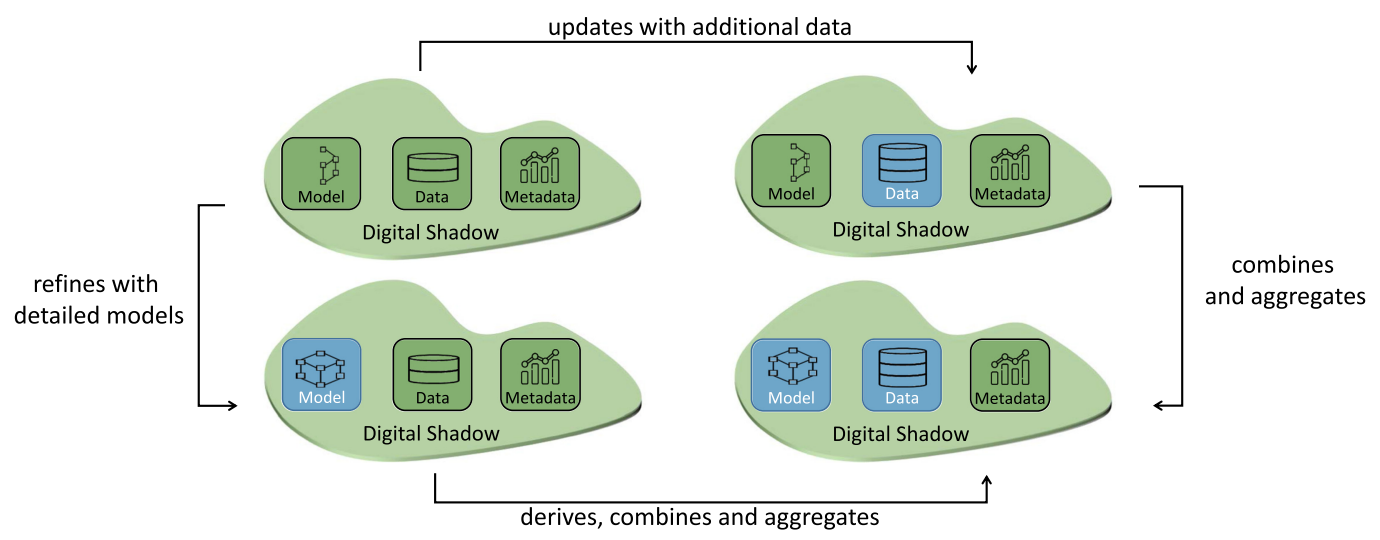

Fig. 3. Beyond data, Digital Shadows contain models and metadata that provide insight into the data's context, traces, and interdependencies.

Digital Shadows are sets of contextual data traces or their aggregation and abstraction collected from a system or mockup, such that they help to fulfill a specific purpose with respect to the original system. They are comprised of data, metadata, and models.

Digital Shadows are created on the fly to be semantically sufficiently correct for their specific purpose. To this effect, they are generated by the application of data analytics and reduced engineering models. The former translate data (in real time) to information (possibly by involving machine learning algorithms), the latter enable relating that information and giving semantics (meaning) in the context of the purpose, e.g., the production system or process the information was produced from. Consequently, digital shadows may contain (parts of) engineering models, simulation models, or other models of the system whose part or activity they represent. Understanding Digital Shadows as interfaces for production services enables re-using these services with refined or abstracted Digital Shadows for subsequent tasks. Thereby, Digital Shadows continuously improve with their usage, since the underlying production models are validated and extended with each additional application. Digital Shadows benefit from interconnected production plants because they can access and be composed of more data from different data sources. With more data available, they can become more meaningful and thus more effective in supporting automation. The technical realization of a Digital Shadow potentially includes different pieces of information but should at least contain (i) the data (or an abstraction thereof) collected in the monitoring period; (ii) metadata, such as period of time, the sensors used, the sampling frequency, potential uncertainties, information about the state of the system during operation (if that is not part of the sensed data), the intention of the measurement, who was involved, and so on; and (iii) contextual data, such as references to the engineering model (e.g., CAD, Simulink, SysML, or UML) of the observed system or process, for instance, describing, where sensors are attached to and thus where measurements are taken from. A Digital Shadow includes the information on how it has been computed as metadata; thus, we know about the constraints and limitations of the dataset. This information is crucial when examining the data quality of a Digital Shadow, i.e., by changing the function that produces the Digital Shadow, we can improve its data quality.

Figure 3 shows the components of Digital Shadows: models, data, and metadata. We refer the reader to our work [10] for details on the conceptual model of Digital Shadows. Digital Shadows refine other Digital Shadows by providing more detailed models, by adding up-to-date data, by 
deriving new Digital Shadows using AI methods, or by combining the information contained in other Digital Shadows into a new Digital Shadow. Individual Digital Shadows not only serve to control individual production processes, but can be linked together within or across companies Combining Digital Shadows across different processes and companies to a WWL delivers added value by providing more information that can reveal additional insights on the production, identifying correlations of subsequent process steps or actors along the value chain, as well as transferring of recognized patterns to similar but new processes.

\section{ESTABLISHING THE WORLD WIDE LAB (WWL): MAKING KNOWLEDGE GLOBALLY ACCESSIBLE}

Digital Shadows are an important aspect when capturing information that was generated as part of the IoP. To realize the main application of the IoP (cf. Section 1), research must also focus on open aspects of the envisioned WWL, i.e., how to securely realize the exchange of knowledge across different stakeholders to enable approaches like transfer learning. The WWL complements Digital Shadows by offering stakeholders the ability to reliably collaborate in an industrial setting and, ultimately, to improve their existing Digital Shadows, thus tapping into currently unrealized potentials.

World Wide Lab (WWL). The WWL connects all (existing) data sources in a globally interconnected system and makes them available across company borders to foster a transfer of knowledge and to fuel innovation. To this end, available information is re-used across all phases of the product cycle, i.e., development, production, and usage. The WWL is not fixed to a single architecture or set of stakeholders. These decisions are use case-specific.

The term WWL is chosen as an analogy to the WWW as the envisioned WWL should also provide (unstructured) information in a large-scale system that is maintained by multiple (distrusting) stakeholders in a similar way as the Internet. Naturally, such a setting also requires in-depth analyses of the underlying security principles and privacy needs to make sure that the new types of dataflows [122] and data sharing concepts are implemented securely [120]. We envision combining data sources from different production sites, supply chains, data lakes, and cyber-physical systems (CPSs). Thus, we make information, potentially provided by competitors, available across company borders to eventually make it accessible within an established WWL. This change in boundaries enables companies to improve their decision-making by combining data sources and Digital Shadows on a larger scale [121]. For instance, comparative process mining using process cubes [146] allows informed decisions by comparing different processes and their properties.

The Need for (Data) Security and Safety. Traditionally, companies in the production domain are cautious when sharing data to prevent any leakage of sensitive information [110]. Hence, the transition from today's local data silos to a globally accessible knowledge base [122] is a significant challenge as valuable intellectual property must be protected accordingly. Similarly, even less sensitive data, such as shipment information, might already expose business relationships to the public. Consequently, the identity of involved companies should be concealed through technical means whenever practical [64]. In addition to data security, safety aspects are relevant as data sharing can have a direct impact on the environment, involved workforce, and the local production site [63]. Safety is paramount when using foreign data, as, e.g., incorrect parameter settings applied to a machine can cause physical damage and, even worse, harm to humans. Here, especially, network security policies should be revised to account for the shift from isolated production networks to the global WWL $[63,120]$. Simultaneously, improvements in this area can also mitigate the individual risks of data leakage. Overall, (data) security and safety are fundamental for the WWL. 
Integrating Data Sources into the WWL. Given that a variety of different systems must be integrated, the required changes to shape the manufacturing industry to the WWL affect different areas of today's production. In production cells, the gathering and sharing of process data of individual CPSs and production processes must be dealt with. Concerning our exemplary car manufacturer, this view corresponds to information about a single assembly step in production. On the shop floor, the Digital Shadows of different production cells can be combined for a single production site. W.r.t. to our example, the data collected here refers to all local production steps and their (inter)connections. Finally, on the WWL layer, data and knowledge of different production sites, potentially even across domains [147], should be available for companies in the WWL [57]. Here, data sources are not limited to companies along a single supply chain. Instead, we also encourage an exchange of information across supply chains to maximize the improvements resulting from exchanged information. A recent survey [155] underlines that even the usually considered scenario of smart supply chains is not yet put into practice. To conclude, data sources from different areas in manufacturing, i.e., production cells, shop floors, and the WWL layer, have to be accessible within the WWL to provide the needed information and variety.

As highlighted before (cf. Section 3), all available data is part of the current state of knowledge, which is not in a fixed state, but in continuous change, as new process information and data ideally help to improve the existing shadows [57]. The car factory could, for example, retrieve machine parameters gathered in a different setting (by another stakeholder) to react to changes in the hardness of the delivered steel. Overall, reaching decisions is more efficient and reliable with the WWL because all globally available knowledge is incorporated into the decision-making process.

Estimating the Impact of the WWL. The fully developed and interconnected WWL serves as the ideal real-world application of the concept of the IoP: a globally accessible knowledge base that combines the information of numerous data sources. Without further research, we are unable to fully tap into the expected potentials. In line with the advances made by large standardization projects, such as Gaia-X [18] or the IDS [111, 112], we realized first prototypes of the WWL to showcase its potential to companies. For example, beyond our formalization on data interoperability [57], we already provide insights into the accountable and reliable data sharing in supply chains $[5,117,118]$. While research traditionally focuses on data sharing along the supply chain, we also particularly explore the data sharing across supply chains, and especially when trust relationships are missing [123]. For example, we revisited the privacy needs in company benchmarking across supply chains and discovered that existing work does not account for the sensitivity of the complex computations of key performance indicators [124]. Using readily-available building blocks from confidential computing, we demonstrate that secure approaches are feasible [124] and serve as candidates for real-world use in industry and the WWL.

Regarding the sharing of production parameters and associated experiences, we analyzed the industry needs when commissioning new production lines and correspondingly developed an oblivious exchange platform to facilitate such information sharing [119]. Again, we rely on well-known concepts from confidential computing to ensure security and real-world deployability in the WWL. With two distinct use cases (injection molding and machine tools), we showcase our platform's universality, i.e., our work is not bound to a specific use case. To conclude, our work proves that turning the WWL into reality is possible with concepts from confidential computing. Additional research is needed to transform novel applications into secure, reliable services in the WWL, which are then re-usable across different domains.

An Outlook into Tomorrow's WWL. In particular, we identify a significant need for future research (also concerning readily-available building blocks) in the area of (federated) privacypreserving machine learning. We expect the WWL to enable such applications on a large scale and thus be a source of advancements. For example, when high-pressure die casting parts for cars, 
machine learning-based quality prediction allows discovering defects even when in-situ methods are not applicable. Enriching the input data with external data would significantly improve the prediction results, allow for properly configured production lines, and thereby reduce scrap rates. Especially when directly feeding information into live processes, safety needs must be considered in light of external knowledge sources with its diverse stakeholders. Naturally, more in-depth collaborations, data exchanges, and novel, currently unexplored use cases and applications will emerge once first experiences have been made [122]: Both the perceived advantages and a decrease in reservation against data sharing due to the fear of data leakage will accelerate this development. For now, our work showcases the WWL's potentials, and our findings contribute to standardization efforts, such as Gaia-X or the IDS, that will eventually manifest the WWL in practice.

In the following, we first mention challenges for the WWL, highlight the progress that has already been made, and then formulate necessary further research directions.

\section{SETTING THE STAGE FOR THE INTERNET OF PRODUCTION}

The last two sections laid out the methodological foundation of our approach of the IoP. Digital Shadows provide purpose-driven collections of data, facilitating data-driven decisions. The WWL connects these Digital Shadows in a global network, paralleling the idea of the web as the prime application of the Internet. By establishing an interconnected knowledge base consisting of data sources from various companies, we achieve massive economies of scale, thus increasing the overall benefits. However, to the same extent, we enable several new hurdles that need to be tackled. In the following, we examine these challenges using the top-down layered model shown on the left in Figure 2. For each layer, we highlight research that has already been started to address these issues. We will point the reader to other publications by the authors in which more concrete research results are reported, such as the usage of Digital Shadows in process mining [22] or adaptation of the production system to the capabilities of the worker [102].

\subsection{Humane Interfaces for Interacting with Digital Shadows}

The new possibilities of the IoP have given rise to new questions in the area of designing the interfaces between the human actors and the IoP that have so far been insufficiently addressed in current research [78]. On the one hand, more and smarter automation raises the question of responsibility and control $[93,133]$, and on the other hand, new forms of hybrid intelligence as the collaboration between human operators and the IoP must be designed that harness the potentials of both artificial and human intelligence [44, 89].

Despite the obvious potential of increasingly automated production control through IoP-based Digital Shadows, people will remain an integral component of socio-technical production systems (STPS) [50, 78]: Either as certain tasks cannot be fully automated for technical, legal, or ethical constraints, because of a shift from manual activities to monitoring and planning tasks, or as a final arbitrator when automated systems fail or come into conflict [150].

However, reliable automation leads to the automation conundrum [46]: The more systems are automated, and the higher the performance of the automation, the lower the supervisors' situational awareness and the more difficult supervision, intervention, or manual control becomes. Thus, several challenges need to be addressed to support operators' interaction with Digital Shadow-based automation at all company levels (e.g., shop floor operation, factory planning, supply chain management, and strategic planning).

Transparent Automation and Meaningful Control. Operators' and decision makers' process knowledge and understanding deteriorate through abstraction and automation [6, 9, 46, 150]. However, this situational awareness is crucial should automation fail, to evaluate the functioning of an automated system, or to handle unmodeled situations (out-of-the-loop loss of situational 
awareness). Consequently, a challenge is to design simple interfaces to automated processes and decision-support systems that are accessible, transparent, and easy to learn and use.

Although one of our intended interfaces is as simple as an Internet search engine (cf. Figure 1), processing these queries is more sophisticated than a simple keyword search. As users should not need deep technical knowledge about the underlying system, autonomous agents interacting on the WWL can provide this semantic information implicitly. For example, when inquiring about the ideal type of an electric car battery, the planned production method or driving safety is considered implicitly. Other forms of automation can draw on transparent explanatory approaches so that the basis for the system's decisions can be interpreted, understood, and corrected if necessary. An emerging research field to increase usability and comprehensibility of models is Explainable AI [4], whose approaches and methods must be adapted to the specific use cases of the production domain.

Modeling of Tacit Knowledge. A further challenge is the utilization of human expertise through automated systems. Most machine learning approaches require representations of human knowledge to create digital models of product and production planning, as well as production and usage. In some cases, the description of this knowledge is simple and often already exists (e.g., image classification for online quality control, if quality can be measured easily). In other cases, a representation of the expert knowledge is necessary, but this tacit knowledge is difficult to verbalize and hidden in unconscious evaluations and motor memory [126]. Consequently, it is difficult to communicate this knowledge and expertise to others and other domains, to record and describe this knowledge digitally, and to use sparse data to train AI algorithms [89].

Bias-Free Interaction with Automated Systems. Trust, reliance, and trustworthiness is a crucial prerequisite for acceptance and use of automation in production and other domains [65]. Adequate and meaningful use of automation by operators must be carefully balanced between disuse (intentional neglect of decision aids, either due to missing trust or missing perceived benefits) and misuse (over-utilization of automation by over-trust and neglecting to check its results) $[65,114]$. This fine balance relates to automation biases and automation complacency, and sound systems design helps $[19,58]$ : if automated systems are designed right, operators have more capacity to detect malfunctioning automation and to handle exceptions.

Context- and User-Centered Interfaces. Third, a challenge is to make the vast amount of heterogeneous information from the IoP transparent and accessible through user-, context-, and task-dependent interfaces [1,29]. Again, the design of STPSs and interface usability are important factors, as good interfaces facilitate the understanding of the systems' status and functioning, lower cognitive load, enable successful operation, and offer insights on further optimizations. Furthermore, we need a "natural and trusted" communicative etiquette for enabling a close and trusted collaboration between the operators and the AI-based systems. For this, understanding the operators' basic emotional needs as well as their mental models of and general attitudes towards these highly complex systems is of importance. Furthermore, the demographic shift and changes in the workforce pose further challenges, as user interfaces and support systems must take older workers and their specific requirements, different skill-sets, interests, and abilities into account [34, 47].

In prior work, we have shown that good user interface design is crucial for successful and trustful interaction with automated production systems [125] and robots interacting closely with humans [14]. Well-designed user interfaces mitigate automation biases by enabling operators to intervene should automation fail [19].

Example. Taking the production of an e-vehicle as an example, the IoP results in changes for workers along the value chain. Through human-centered design of decision dashboards and approaches such as Explainable AI, decision support systems can provide transparent suggestions for improving the performance of production processes, quality insurance, or the supply chain, thus reducing human errors in decision making [19]. Also, by continuously capturing the 
interactions of experienced workers with the production systems, their knowledge can be integrated to improve future recommendations for novices. Furthermore, digital images of the workers' capabilities and requirements can be generated and used to orchestrate the collaboration between production systems and workers, for example, by adopting the speed of production process and human-robot collaboration to the workers' needs [102].

Takeaway. Overall, the adequacy of these systems and their design should not be determined by experts from engineering, computer science, or ethics alone, but rather in partnership with the employees. A participatory design ensures that the technological advances and implementations of STPSs are harmonized with people's capabilities, norms, and values.

\subsection{Model-Integrated Artificial Intelligence with Autonomous Agents in the IoP}

Within the IoP, the goal is to create a synergy between data-driven AI methods and state-of-the-art model order reduction techniques from engineering mathematics across disciplinary boundaries. This enables a high level of automation to realize real-time decisions, through built and shared Digital Shadows. E.g., in e-vehicle manufacturing, sheet metal is still a fundamental material whose processing consumes large amounts of energy, while deviations are safety-critical. Therefore, integrating reduced engineering models of material properties with machine learning in the hot rolling process to inform artificial networks as Digital Shadows enables real-time compensations for deviations during the process [103]. This adjustment allows significant energy savings. For this degree of automation, which is needed to gain all data and knowledge from different sources and domains worldwide to build Digital Shadows and realize model-integrated AI, we need autonomous agents [131] based on various AI techniques like knowledge-based systems or machine learning to name but a few. Manual data queries that would otherwise be required would be infeasible given the level of cross-domain collaboration and networking.

Another example in e-vehicle manufacturing covers sophisticated logistics robots used in modern modularized factories without assembly lines [25]. In such complex settings, sophisticated logistics robots controlled by autonomous agents can help to assemble products, integrating different AI methods $[66,67]$. Similar methods were used for autonomous agents communicating as programs with the WWW to realize Semantic Web applications [100, 127]. In addition, further examples of successful applications of agent technologies in industrial settings exist [90, 91].

In the IoP, we develop autonomous agents, called WWL Agents [21], in a multi-agent network connected to information sources for semantic information, e.g., ontologies [99] and knowledge graphs providing provenance information [57]. With the latter, it is possible to get, e.g., the origin of data used for training an artificial neural network representing a particular Digital Shadow or the usage history of mathematical models comprised within the Digital Shadow to solve difficult production steps.

Comparable agent systems in the literature are very often only used to support manufacturing processes within a single production facility or company [75, 90, 91]. The novelty of our approach is that the purpose of WWL Agents is realizing interoperability in the WWL and breaking data silos enabling data-intensive AI approaches, such as machine learning, to generate specific Digital Shadows. For instance, communicating with agents from other companies, apply different AI methods for sharing, generating, and using their data and Digital Shadows from different production domains is their main function. Furthermore, with semantic information about the origin of the data, they can provide detailed information of solutions found by a WWL Agent to users around the world. In addition, this approach can incorporate existing local multi-agent production systems if they provide an interface to the WWL.

Explainable AI. To be able to present comprehensible results for humans as discussed above, algorithms need to be able to explain why their results are reasonable and accurate. Such 
explainable AI methods $[2,45]$ can be provided by knowledge-based systems because they represent the knowledge in a human-understandable way [17]. However, one of the challenges is to find the right explanations and their representation for production processes. Furthermore, other AI approaches as, e.g., machine learning or process mining [3], need to be included in trustworthy explanations.

Furthermore, in the IoP, semantic information about the data in the WWL is available to calculate appropriate answers. Given that the IoP presents a well-understood domain [20], the semantic information is already represented in existing models and methods. However, it is often not machine-readable and not yet linked to the raw machine-produced sensor data, which need to be exploited by software agents. Open questions are how to gain machine-readable semantic information from the existing models and how to link the semantic information from the engineering models to the data to build trustworthy software agents [141] dealing with the semantics in Digital Shadows and supporting the decision process of engineers in the WWL.

WWL Agent Dialogs. In the vision of the IoP, WWL Agents realize user dialogs via interfaces like shown, e.g., in Figure 1, and help the users to get the appropriate answers to their problem in the sense that it improves the product and the production processes. These agents need to be interconnected within the WWL to enable them to integrate information from external data sources as well. With this information, the agents are able to compute solutions or suggestions and generate answers to the user requests. Here, the challenges lie in realizing a human-machine communication that is understandable for the humans working in the production. Additionally, the relevant information from the WWL has to be identified to give adequate answers, which really lead to process improvements.

Multi-Agent Network. We claim that only the WWL delivers the amount and variation of data that is necessary to build Digital Shadows as, e.g., trained artificial neural networks, so that they can provide aids for specific purposes in a production process. Therefore, WWL Agents in a world-wide multi-agent network are a key factor in reaching this level of interoperability.

By communicating via the WWL, the agents share services, data, and knowledge, so that other agents can support their local clients with their production. In that way, it is possible, for instance, to realize a fully automated on-demand pull production [70], with implications along the whole supply chain, as long as every participant is connected to the WWL. Thus, production can dynamically adapt to local or global changes, such as product design modifications, local production failures, or supply chain variances in case of a strike, natural disaster, or pandemic. The challenges range from finding the appropriate network structure for the agents to how requests to agents are processed.

Standardized and Open Communication Protocols. Similar to the WWW, the WWL can only unfold its full function if a crucial number of participants is able to share their information in the network. The success of the WWW was only possible because there was an open access to all its protocols as HTTP. Therefore, for the IoP we intend to let the autonomous agents use protocols based on HTTP and other open standards. Furthermore, new protocols which are needed for the communication between the autonomous agents have to be freely available and standardized in the long run to let everyone participate in the WWL with their own agent.

Connection to Versioned Data Storages and Ontologies. The agents in the WWL have to be connected to various information sources to gain the knowledge they need to give proper answers to the user. For doing so, the agents need semantic information about the posed queries. Therefore, we want to use semantic techniques, for example, ontologies [116] and versioned knowledge graphs providing provenance information about data, models, and knowledge from production processes [57]. With the former, the agents gain semantic information about the terms which are used in user requests. With the latter, the agent can get provenance information about the origin 
of production data or the history of a product part. Here, challenges are, for instance, how the semantic information can be used and how the information from different data sources can be combined to improve production processes.

Example. Assume a setting where an e-vehicle manufacturer wants to improve the material choice for the main car body. Then the engineer, e.g., instructs a WWL Agent to first collect information about available steel composites from different steel producers and then analyze them according to the characteristics of the local production process as described by a Digital Shadow. This Digital Shadow could be created by another agent in the WWL analyzing the processes in the car factory and using AI methods such as machine learning. Provided with semantic information about the processes and the materials which are planned to be used, a WWL Agent can give the user a detailed answer with explanations and links to the provenance of the agent's information.

Takeaway. The integration of (mathematical) models from engineering with AI models enables new opportunities, and makes WWL Agents the enabling factor in the IoP. To this end, they rely on the connection to various data sources from different production companies, and standardized protocols. Existing knowledge-based agent technology needs further extensions to meet the demands of the manufacturing industry in the setting of the IoP.

\subsection{Model-Driven Digital Shadows}

The term model-driven refers to development methodologies that rely on abstract models of systems as central development artifacts [148]. These models carry explicit domain expertise and serve as a foundation for communication, documentation, analysis, and synthesis in agile development projects [129]. They can be systematically transformed into concrete implementations [49] such as Digital Twins [13, 43], privacy-preserving IoT systems [104], information systems [54], or assistive systems [105]. Digital shadows [88, 128, 135] relate to models, can carry models themselves, and serve as (aggregated) abstractions of models for automated processing [13, 43].

Cross-Domain Collaboration. Interdisciplinary teams consisting of experts from the production domain, computer science, automation, and many more develop a new generation of CPPSs. All of them contribute individual expertise, perspectives, paradigms, technologies, and solutions to the IoP. And often, this expertise is encoded in different kinds of models [69, 105, 152]. The successful and efficient integration of domain-specific knowledge into the IoP is crucial to construct the multi-perspective data and models at design time, simulation time, and run time. In our vision, Digital Shadows also serve to semantically enrich process data to enable (automated) decision making in (domain-specific) real time. To this end, they must be semantically integrated with data and models engineered during design and simulation [80]. This need demands a modeling of detailed aspects (from manufacturing system details to factory behavior, to strategic goals, to interface descriptions) in sufficiently formal languages $[129,130]$.

Systems Engineering. Model-driven systems engineering [12, 42] lifts models to primary development artifacts that increase abstraction and engineering efficiency in the interdisciplinary engineering of cyber-physical (production) systems. These models usually conform to (domainspecific) modeling languages (such as Simulink [37], SysML [51], or AutomationML [98]), that provide experts with required functionality and facilitate describing and integrating systems engineering concerns. Consequently, the system description is distributed over several models and tools that currently are not syntactically and semantically integrated. Designing and engineering the systems of the IoP, therefore, demands novel solutions for the automated, ad-hoc integration of modeling languages and their tools, e.g., as presented by Dalibor et al. [41], such that experts of the different domains can leverage modeling views tailored to their desired level of abstraction across domain boundaries and optimized for analysis. Software Language Engineering (SLE) [68] is a discipline that investigates the efficient engineering and integration of heterogeneous modeling 
languages; hence SLE is a crucial prerequisite for providing domain-specific representations and integrating knowledge from various domains within the IoP.

Integrating Modeling Languages and Tools. This model and language diversity [152] will also be reflected in Digital Shadows that need to provide optimized structures for handling large amounts of data, selected engineering models, formalized knowledge about data, models, and their context. To this end, Digital Shadows need to be able to integrate high-volume structured and unstructured data with semantically rich, detailed engineering models, and knowledge bases. Thus, we need techniques capable of linking the different underlying modeling languages $[27,138]$ at system design time as well as ad-hoc at runtime. These techniques consider syntactic [62] and semantic integration [33] to bridge semantic gaps between the languages used to express parts of the Digital Shadows, as, e.g., realized for the design of experiments in injection molding [13]. A first concept on how Digital Shadows can be created and are handled during runtime together with process mining techniques has been derived [22]. Efficiency is crucial to also enable an efficient application to very large models. Therefore, research should leverage techniques from database schema modeling [76] and artificial intelligence to enable compositional mechanisms [28] for syntactic and semantic abstraction, aggregation, and integration of data and models.

Example. Domain experts from e-vehicle production define one or more Digital Shadow Types [22] based on a conceptual model [10] for purposes related to production, e.g., quality monitoring or predictive maintenance. These types can be used as blueprints for concrete Digital Shadows recorded from production data at runtime, e.g., a Digital Shadow Type might serve the purpose to minimize the product rejection rate of the grinding process of front window panes and capture the related information accordingly. During runtime, the Digital Shadows are created according to their types and populated with models, data, and metadata. Using such Digital Shadows, the rejection rates from every window pane are aggregated to every job on a grinding machine. Periodically, a new Digital Shadow is created that aggregates again the rejection rates based on the new time slice.

Takeaway. Purpose-driven Digital Shadows can be created and provided at runtime using design time models; thus, model-driven development supports and simplifies the automation of production.

\subsection{Interconnected and Industry-Capable Infrastructure}

The concept of Digital Shadows is based on the notion that a problem-specific view on the overall process can be derived from a sufficient amount of process-related data. To this end, process data needs to be recorded and collected, ideally in a fine-grained manner and by a variety of different sensors, to provide a comprehensive description of the process. Subsequently, this description can be scaled down to match the concrete requirements of a specific problem or task. In general, the quality of the Digital Shadows correlates with the quality and the richness of the available data, i.e., larger amounts of data are generally favorable. Companies are thus incentivized to collect, process, and store huge volumes and varieties of data, which consequently requires a capable infrastructure. Setting up this infrastructure and enabling the global WWL and its models introduce several obstacles.

Data Integration. Availability and accessibility of information with high data quality is an important issue in many production and business processes. For example, the quality assurance of production companies could require access to detailed process data a long time after the production is finished, which cannot be realized by traditional data integration approaches that use carefully engineered data processing workflows to extract, transform, and load data into an integrated data store. For the collection of data, we envision a data lake platform in which data is stored in its raw format without prior integration or aggregation [61, 74]. Compression techniques on sensor data could be applied to address the real-time requirements by reducing the amount of data, but a 
lossless compression should be guaranteed. For example, in a use case of Laser Powder Bed Fusion, in which high power-density lasers are used to melt and fuse metallic powders, we apply dynamic compression techniques to reduce the data volume, but to maintain the information content.

The data lake stores raw data to avoid restricting the data analysis to a predefined integrated schema. The data in the lake should be enriched with semantic metadata and data quality information (e.g., source, accuracy, and time) to make it interpretable and usable in various applications.

Data Collection. As the data lake is intended to collect information from many sources in the WWL, the underlying infrastructure must be able to transfer very large amounts of data. This requirement is independent of whether the data lake is deployed on-premise or in the cloud.

We already identified that cumulative data rates for a single production cell can easily be in the range of giga- to peta-bytes for settings where several machines are interconnected [55]. Directly transmitting all data is thus often infeasible as available data rates are too low. The current bandwidth limitations dictate pre-processing and aggregation to make the WWL possible at all, although this form of data reduction techniques technically contradicts our previously stated requirement that data lakes should obtain all information. Thus, it is vitally important to devise domain knowledge-based methods which can first reduce the amount of data that needs to be transferred without loss of information, e.g., if some values can be derived from others. In this context, Lipp et al. [96] propose a process-driven data collection that allows to statically configure which data needs to be collected in which phase of the process at what granularity. This high level of control allows to precisely adjust the amount of generated data to the required signal accuracy as well as the available bandwidths.

Similarly, compute capabilities in the network can also be used to first dynamically detect the current process phase (opposed to the static definition by Lipp et al.) and then scale the generated data volume as needed, e.g., ensuring high data quality in times of interesting process behavior while reducing the load in idle times [87]. Additionally, these in-network processing techniques allow for handling data at line-rate and can thus more easily further reduce the load by removing (presumed) irrelevant information or by performing pre-computation steps [55]. Finding the right trade-off between storing as much raw data as possible while also adhering to infrastructure limitations by reducing the transmitted data is currently a predominant challenge. In the aforementioned use case of Laser Powder Bed Fusion, for example, the compression techniques automatically adapt their configuration to the network bandwidth, processing capabilities, and data structures. Provisioning a suitable infrastructure with sufficient bandwidth and storage capabilities is certainly a long-term goal, enhanced by carefully placed and designed in-network compute functions.

Low-Latency Guarantees. Apart from high data rates, the IoP-enabling infrastructure must also satisfy tight latency bounds, which, for example, are needed for process control [132]. This constraint is particularly relevant if decisions are to be made by a remote system or individual based on broader information from the data lake, rather than by process-near controllers solely based on local knowledge. In this case, physical latencies between the processes and remote systems are often already too high for very time-critical applications (sometimes with requirements in the one-digit millisecond range), rendering pure remote solutions, e.g., over the Internet, infeasible. In-network processing again offers a solution as control programs can be deployed in the network and thus significantly reduce the inherent latencies. These programs currently range from simple LQR controllers [132] to basic line detection mechanisms [56] and can thus cover a variety of simple control tasks. Additionally, complementary safety measures, such as emergency stops, can also be realized in networking hardware, as is demonstrated by Cesen et al. [31]. The accuracy and computing speed of such approaches is generally capable of reaching levels similar to userspace applications, while networking devices are especially capable of processing significantly 
higher packet rates [86]. Yet, implementing the required functionality on the current generation of programmable networking hardware is still challenging [86].

Control Loops. Thus, in the context of the IoP, we envision that critical decisions for cyberphysical control loops are made quickly within the production cell, e.g., emergency shutoffs for safety reasons. More complex controls are then implemented at the edge, within the network (innetwork processing), or in the cloud, where they can source additional data sources. Consequently, the (local) decision quality improves with an IoP. Due to the interconnected nature of manufacturing processes, companies even have the chance to account for issues in subsequent process steps. For example, they can react to slight deviations or inaccuracies with control loop adjustments in the next production cell, effectively implementing a real-time control loop for the shop floor. Here, companies can rely on well-known approaches, or, in line with the IoP, they can utilize control loop adjustments that originate from model-integrated artificial intelligence and the knowledge base in form of the WWL.

Device Heterogeneity. The immense heterogeneity regarding the involved sensors and machinery, which often characterizes industrial production settings, is another challenge that needs to be addressed $[1,35]$. For example, depending on the vendor, sensors might differ in their expressiveness or in the protocols that they support. Addressing this challenge on a system user's level, Bodenbenner et al. [16] propose a domain-specific language that abstracts from the sensorspecific details and allows a unified access to the sensor information. However, their solution does not directly address how such sensors can actually be integrated and interconnected on a technical level. Additionally, devices can also change dynamically, e.g., if a process is reconfigured to allow for the production of different products. High flexibility is thus one of the most important infrastructure requirements. Especially when considering the long lifetime of industrial devices, protocols capable of providing security even in these heterogeneous settings are needed [40]. Paniagua et al. [113] provide a survey of different architectural frameworks for Industry 4.0, such as FIWARE, IDS [112], or BaSys4.0. Although these frameworks also address device heterogeneity, they are not specific on the level of communication protocols. More specific digital industrial platforms are provided by different Industry 4.0 key players such as Siemens (MindSphere) or Bosch (IoT Suite) [115]. However, these platforms are often customer-specific solutions, i.e., they claim to address heterogeneous data and devices, but often require a significant customization effort to fit the needs of specific use cases. Additionally, we note that despite the trends of security-by-design and privacy-by-design, devices and protocols must be configured correctly to benefit from these, which is, however, a frequently neglected aspect [38, 39].

Example. Overall, an interconnected and industry-capable infrastructure will enable the evehicle manufacturer to move away from a traditional assembly line towards a more dynamically reconfigurable sequence of production steps [25]. In this context, the flexible data collection and integration centered around the data lake is key for ensuring a sufficient richness of data even in such dynamic settings; thus, allowing access to detailed process information at all times, e.g., for quality assurance purposes. While technical advancements, e.g., regarding storage and data rates, are important to steadily increase the amount of processible data, layered control loops will enable a fine-grained control of all running processes to improve the overall efficiency of the system and allow for quick responses to system changes.

Takeaway. The specific characteristics of industrial environments pose challenges that cannot be addressed by traditional data management and networking solutions, as some aspects require the inclusion of remote services or edge computing while other aspects simply do not support such approaches. Instead, a concept is needed, which carefully includes mechanisms to satisfy all of the mentioned needs: high-data rates, support for heterogeneous data structures, low-latency data processing, and flexibility. 


\begin{tabular}{|c|c|c|c|c|c|}
\hline Layers Challenge & $\begin{array}{c}\text { Standardized (Data) } \\
\text { Interfaces }\end{array}$ & $\begin{array}{c}\text { Interconnecting } \\
\text { (Domain) Knowledge }\end{array}$ & $\begin{array}{c}\text { Burden-Free } \\
\text { Operation }\end{array}$ & $\begin{array}{l}\text { Real-World } \\
\text { Integration }\end{array}$ & $\begin{array}{c}\text { Long-Term } \\
\text { (Information) Usage }\end{array}$ \\
\hline $\begin{array}{l}\text { Humane } \\
\text { Interfaces }\end{array}$ & $\begin{array}{l}\text { Context- and User- } \\
\text { Centered Interfaces }\end{array}$ & $\begin{array}{c}\text { Facilitate Transfer } \\
\text { of Expertise }\end{array}$ & $\begin{array}{l}\text { Bias-Free } \\
\text { Automation }\end{array}$ & $\begin{array}{l}\text { sparent } \\
\text { imation }\end{array}$ & $\begin{array}{l}\text { Modeling of Tacit } \\
\text { Knowledge }\end{array}$ \\
\hline $\begin{array}{l}\text { Autonomous } \\
\text { Agents }\end{array}$ & $\begin{array}{l}\text { Standardized } \\
\text { Communication } \\
\text { Protocols }\end{array}$ & & $\begin{array}{c}\text { WWL } \\
\text { Agent Dialogs }\end{array}$ & & $\begin{array}{l}\text { Connection to } \\
\text { Versioned Data Stores } \\
\text { and Ontologies }\end{array}$ \\
\hline $\begin{array}{l}\text { Model-Driven } \\
\text { Digital Shadows }\end{array}$ & $\begin{array}{l}\text { Integrating Modeling } \\
\text { Languages and Tools }\end{array}$ & Engineering & $\begin{array}{l}\text { Domain-Specific } \\
\text { Representation }\end{array}$ & $\begin{array}{l}\text { Interface } \\
\text { Description }\end{array}$ & $\begin{array}{l}\text { Cross-Domain } \\
\text { Collaboration }\end{array}$ \\
\hline $\begin{array}{l}\text { Interconnected } \\
\text { Infrastructure } \\
\text { \& Data }\end{array}$ & $\begin{array}{c}\text { Device } \\
\text { Heterogeneity }\end{array}$ & $\begin{array}{r}\text { Data } \\
\text { Integrati }\end{array}$ & $\begin{array}{l}\text { Low-Latency } \\
\text { Guarantees }\end{array}$ & $\begin{array}{c}\text { Data } \\
\text { Collection }\end{array}$ & $\begin{array}{l}\text { Persistent } \\
\text { Identifiers }\end{array}$ \\
\hline
\end{tabular}

Fig. 4. Matrix of strategic research directions and layers in the loP. The size of the blue circles corresponds to the priority (the bigger the more important).

\section{STRATEGIC RESEARCH DIRECTIONS FOR THE INTERNET OF PRODUCTION}

The challenges discussed above show that a sustainable transition towards smart industrial production within the framework of Industry 4.0 is necessary that goes well beyond an interdisciplinary collaboration between engineers and computer scientists. Several disciplines are being involved in computer science, as we have demonstrated with the level-based construction of arguments in the previous section. While we have so far approached the challenges individually by computer science disciplines, we are now looking at a comprehensive view of necessary research efforts. To this end, in the following, we present five general research areas in the domain of computer science that need to be addressed to turn the concept of the IoP into reality. Figure 4 gives an overview of the research areas and the required research efforts.

Standardized (Data) Interfaces. First, to ensure compatibility between individual components, standardized interfaces, and communication protocols are necessary. This is an elementary prerequisite for a large-scale infrastructure in which heterogeneous data sources and vendor-specific peculiarities participate. The development of a single all-embracing formalism is unrealistic. Instead, mechanisms for linking and mapping between different data sources, models, and systems need to be developed. These mechanisms finally enable the linking of the current information silos. To be able to process queries over such linked data sets, the query processing mechanisms must take mappings into account and should be able to retrieve and to reconcile data from different sources. Model-based systems can facilitate the aggregation of heterogeneous systems. These systems, in turn, help autonomous agents to communicate with each other and with the devices in the WWL via standardized interfaces. Thereby, the knowledge representation in the IoP should also be standardized. As the last element, interfaces to humans need to be included here, which can, for example, be normalized or specifically adapted for the usage context. Ultimately, the concept of digital shadows must be further refined here, as their combination with humans can form an exceptionally smart joint cognitive system. The domain knowledge leads us to the next aspect.

Interconnecting (Domain) Knowledge. The interconnected domain knowledge must be shareable and accessible to contribute to the global knowledge base. It must be accessible by autonomous agent networks, as well as operators that want to build on the knowledge from other domains. To this end, mechanisms and models must be developed, to allow integration and provision of metadata and context together with the data. Today, most research on security and privacy 
is focused on specific human-specific data. To enable secure collaboration in competitive industrial scenarios, appropriate technical solutions are needed, with a special emphasis on the area of connecting previously unaffiliated businesses. Here, questions arise that look into the privacypreserving matching of relevant resources with an inquiring party. The goal should be to create a distributed decentralized knowledge service that operates on data in the WWL.

Burden-Free Operation. Some hurdles need to be overcome on the path to a world-scale deployment. A burden-free operation ensures that companies are interested in connecting to the IoP and integrate it into their production sites. Therefore, an effortless implementation must be ensured. Model-based approaches need to be seamlessly integrated to allow autonomous information sharing for triggered queries, answered by autonomous agents. Hereby, it is essential that automation without human intervention works flawlessly and that automated support systems with a human-in-the-loop are designed right to avoid automation bias and the development of mistrust. The infrastructure needs to ensure low-latency guarantees, despite the underlying unstructured peer-to-peer topology. Likewise, real-time constraints mandate automated protocols for bargaining, information retrieval, and exchange, as well as for billing. Consequentially, the system must be adaptable for a diverse set of specific use cases.

Real-World Integration. The large amount of available production scenarios poses a challenge to the real-world integration of the IoP. Even more, within these scenarios, the vast amount of data from heterogeneous sources and their representation in interfaces, as well as their management, are open concerns. In particular, methods for capturing and integrating expertise are missing, especially on the scale of industrial knowledge and data rates. They are crucial to provide sufficient input for the AI component of the production queries. At the same time, operation on the data needs to be transparent to involved humans, including aspects such as explainable AI. Key questions evolve around what information is relevant and how it can be made accessible, transparent, and actionable in a meaningful way, while preserving data correctness [117], to maximize the benefits. With regard to the transition of existing systems, some research opportunities arise. On the one hand, existing model-based representations of expert knowledge need to be re-used. On the other hand, current data silos need to be added into a distributed data storage infrastructure, to enable long-term gains.

Long-Term (Information) Usage. Finally, the last aspect of our research roadmap towards the IoP is long-term usage. Once the required infrastructure is in place, it needs to be flexible enough to be sustained for future generations of industrial production systems. Research on how to implement accountability [118,123] and provenance [57] information in scenarios with wildly-branched dependencies and origins is still in its infancy. Thereby, versioned data is required for agents for consecutive information and explainability. Regarding humane aspects, modeling of tacit knowledge and its digital capture needs to be enabled for the long run. Guarantees for data quality and derived models must be defined to enable not only the usage of data but also to support later reusability. Hereby, feedback and recovery mechanisms need to ensure the connection between models and data, in a permanent, traceable, and synchronous manner. Related to this is defining persistent identifiers for models, data, metadata, and other objects [59]. Further aspects requiring research are long-term reliability of data storage and derived decisions. Similarly, means for conflict resolution need to be integrated into the IoP; only then, the risks for querying and utilizing the IoP are mitigated.

Next Steps. To arrive at the envisioned global IoP, the mentioned research areas have to advance simultaneously and in close collaboration. Hence, experts with suitable interdisciplinary backgrounds are essential. In its entirety, the true value of the WWL as the IoP's prime application will increase through the connection and commitment of as many participants as possible. 


\section{CONCLUSION}

Our vision of the IoP and its enabling concepts of the WWL and Digital Shadows extend beyond singular CPS and aim at the complete vertical and horizontal integration of production systems, smart data analytics, autonomous agents, and task-, context-, and user-adaptive interfaces.

To implement this vision and to realize significant improvements in production, numerous activities in multiple domains are necessary. In this article, we focused on research areas originating from computer science: Production systems must be securely interconnected between different locations, heterogeneous data streams from various systems must be captured, processed, aggregated, stored with a sufficient level of detail, and semantically enriched. An infrastructure for synchronous and asynchronous access to the data must be developed, existing data analysis methods must be applied, and new algorithms that fit the data and processes must be designed. Queries are not necessarily user-defined as concepts from AI should be implemented to establish new connections between the collected data and derived Digital Shadows. Finally, the results of these analyses must be transparently communicated to users of the IoP.

The changes we propose do not have to be implemented overnight, nor can they be. Instead, the IoP allows for a phased approach. For example, Retrofitting is a suitable approach, which acknowledges existing asset-heavy long-term investments by upgrading them with sensors and actuators to get smart production systems [82]. This also effectively addresses the issue of sustainable production systems [142]. In further enhancement steps, initially limited WWL agents can then integrate further AI tools as long as open interfaces are available.

Short-term benefits of the IoP result from higher production efficiency through smarter production control for faster product and innovation cycles, that learns to adapt to new materials and products, and interfaces that make production states transparent. In the long term, the WWL as an application of the IoP will improve production engineering research, increase the viability of industrial manufacturing, and positively impact society and the environment as a whole: By uncovering previously unknown relationships along and across process chains, through the identification of new potential to optimize the production efficiency, energy consumption, and value creation, and by designing interfaces that increase employee autonomy, integrate their capabilities, and are aligned with their values.

Beyond process chains in manufacturing, further implications up to the management level of companies are to be expected by the possibilities the IoP offers. The flexibility unlocked through new data streams will also impact business models. For example, new, dynamic forms of collaboration in corporate networks through data-driven platforms will emerge, extending the research scope from engineering, and computer science to economic disciplines.

We conclude that many complex research challenges still remain to be solved to realize the vision of a trusted, interconnected, and intelligent production landscape. However, the work towards the IoP requires an overarching commitment to provide measurable benefits for industry, research, and society. Thus, to turn the vision of increasingly networked, smarter, and sustainable industrial production into reality, all aspects and their comprehensive and interwoven effects must be well-aligned and understood deeply. Conversely, this requires large, heterogeneous, and interdisciplinary teams led by an integrated research framework. We intend to contribute to achieving these goals within our ongoing research cluster IoP, and invite everyone within computer science to join us in contributing research towards the challenges laid out in this article.

\section{ACKNOWLEDGMENTS}

The authors would like to thank the anonymous reviewers and the editor for their valuable feedback and comments. 


\section{REFERENCES}

[1] Mohammad Aazam, Sherali Zeadally, and Khaled A. Harras. 2018. Deploying fog computing in industrial internet of things and industry 4.0. IEEE Transactions on Industrial Informatics 14, 10 (2018), 4674-4682. DOI : https://doi.org/10. 1109/TII.2018.2855198

[2] Amina Adadi and Mohammed Berrada. 2018. Peeking inside the black-box: A survey on explainable artificial intelligence. IEEE Access 6 (2018), 52138-52160. DOI : https://doi.org/10.1109/ACCESS.2018.2870052

[3] Jan Niklas Adams, Sebastiaan J. van Zelst, Lara Quack, and Kathrin Hausmann, et al. 2021. A framework for explainable concept drift detection in process mining. In 19th Int. Conf. on Business Process Management (BPM'21), Vol. 12875. Springer, 400-416. https://doi.org/10.1007/978-3-030-85469-0_25

[4] Alejandro Barredo Arrieta, Natalia Díaz-Rodríguez, Javier Del Ser, Adrien Bennetot, Siham Tabik, Alberto Barbado, Salvador García, Sergio Gil-López, Daniel Molina, Richard Benjamins, Raja Chatila, and Francisco Herrera. 2020. Explainable artificial intelligence : Concepts, taxonomies, opportunities and challenges toward responsible AI. Information Fusion 58 (2020), 82-115. DOI : https://doi.org/10.1016/j.inffus.2019.12.012

[5] Lennart Bader, Jan Pennekamp, Roman Matzutt, David Hedderich, Markus Kowalski, Volker Lücken, and Klaus Wehrle. 2021. Blockchain-based privacy preservation for supply chains supporting lightweight multi-hop information accountability. Information Processing \& Management 58, 3 (2021), 102529. DOI: https://doi.org/10.1016/j.ipm. 2021.102529

[6] Lisanne Bainbridge. 1983. Ironies of automation. Automatica 19, 6 (1983), 775-779. DOI : https://doi.org/10.1016/00051098(83)90046-8

[7] Eric Ballot, Benoit Montreuil, and Russell Meller. 2014. The Physical Internet. La Documentation Française.

[8] Barbara Rita Barricelli, Elena Casiraghi, and Daniela Fogli. 2019. A survey on digital twin: Definitions, characteristics, applications, and design implications. IEEE Access 7 (2019), 167653-167671. DOI : https://doi.org/10.1109/ACCESS. 2019.2953499

[9] Gordon Baxter, John Rooksby, Yuanzhi Wang, and Ali Khajeh-Hosseini. 2012. The ironies of automation: Still going strong at 30?. In Proceedings of the 30th European Conference on Cognitive Ergonomics. ACM, 65-71. https://doi.org/ $10.1145 / 2448136.2448149$

[10] Fabian Becker, Pascal Bibow, Manuela Dalibor, Aymen Gannouni, Viviane Hahn, Christian Hopmann, Matthias Jarke, Moritz Kröger, Johannes Lipp, Judith Maibaum, Judith Michael, Bernhard Rumpe, Patrick Sapel, Niklas Schäfer, Georg J. Schmitz, Günther Schuh, and Andreas Wortmann. 2021. A conceptual model for digital shadows in industry and its application. In Proceedings of the 40th International Conference on Conceptual Modeling, Vol. 13011. Springer, 271-281. DOI : https://doi.org/10.1007/978-3-030-89022-3_22

[11] Paolo Bellavista and Alessio Mora. 2019. Edge cloud as an enabler for distributed AI in industrial IoT applications: the experience of the IoTwins project. In Proceedings of the 1st Workshop on Artificial Intelligence and Internet of Things, Vol. 2502. CEUR-WS, 1-15.

[12] Luca Berardinelli, Alexandra Mazak, Oliver Alt, Manuel Wimmer, and Gerti Kappel. 2017. Model-Driven Systems Engineering: Principles and Application in the CPPS Domain. Springer, 261-299. DOI : https://doi.org/10.1007/978-3319-56345-9_11

[13] Pascal Bibow, Manuela Dalibor, Christian Hopmann, Ben Mainz, Bernhard Rumpe, David Schmalzing, Mauritius Schmitz, and Andreas Wortmann. 2020. Model-driven development of a digital twin for injection molding. In Proceedings of the 32nd International Conference on Advanced Information Systems Engineering, Vol. 12127. Springer 85-100. https://doi.org/10.1007/978-3-030-49435-3_6

[14] Hannah Biermann, Philipp Brauner, and Martina Ziefle. 2021. How context and design shape human-robot trust and attributions. Paladyn, fournal of Behavioural Robotics 12, 1 (2021), 74-86. DOI : https://doi.org/10.1515/pjbr-2021-0008

[15] Florian Biesinger, Davis Meike, Benedikt Kraß, and Michael Weyrich. 2019. A digital twin for production planning based on cyber-physical systems: A case study for a cyber-physical system-based creation of a digital twin. Procedia CIRP 79 (2019), 355-360. DOI : https://doi.org/10.1016/j.procir.2019.02.087

[16] Matthias Bodenbenner, Mark Pascal Sanders, Benjamin Montavon, and Robert H. Schmitt. 2020. Domain-specific language for sensors in the internet of production. In Proceedings of the 10th Congress of the German Academic Association for Production Technology, Vol. 20. Springer, 448-456. DOI : https://doi.org/10.1007/978-3-662-62138-7_45

[17] Ronald J. Brachman and Hector J. Levesque. 2004. Knowledge Representation and Reasoning (1st ed.). Morgan Kaufmann. DOI : https://doi.org/10.1016/B978-1-55860-932-7.X5083-3

[18] Arnaud Braud, Gaël Fromentoux, Benoit Radier, and Olivier Le Grand. 2021. The road to european digital sovereignty with gaia-x and IDSA. IEEE Network 35, 2 (2021), 4-5. DOI : https://doi.org/10.1109/MNET.2021.9387709

[19] Philipp Brauner, Ralf Philipsen, André Calero Valdez, Martina Ziefle, and Ralf Philipsen. 2019. What happens when decision support systems fail? - The importance of usability on performance in erroneous systems. Behaviour \& Information Technology 38, 12 (2019), 1225-1242. DOI : https://doi.org/10.1080/0144929X.2019.1581258 
[20] Christian Brecher, Sabina Jeschke, Günther Schuh, Susanne Aghassi, Jens Arnoscht, Fabian Bauhoff, Sascha Fuchs, Claudia Jooß, Wilhelm Oliver Karmann, Stefan Kozielski, Simon Orilski, Anja Simone Richert, Andreas Roderburg, Michael Schiffer, Sebastian Stiller, Johannes Schubert, Florian Welter, and Stefan Tönissen. 2012. Integrative Production Technology for High-Wage Countries. Springer, 17-76. DOI : https://doi.org/10.1007/978-3-642-21067-9_2

[21] Florian Brillowski, Lars Gleim, Martin Liebenberg, Thomas Schemmer, Liam Tirpitz, Stefan Decker, Gerhard Lakemeyer, Martina Ziefle, Christoph Greb, and Thomas Gries. 2021. Towards a continuously improving composites manufacturing by employing the internet of production. In Proceedings of the Composites and Advanced Materials Expo Conference.

[22] Tobias Brockhoff, Malte Heithoff, István Koren, Judith Michael, Jérôme Pfeiffer, Bernhard Rumpe, Merih Seran Uysal, Wil M. P. van der Aalst, and Andreas Wortmann. 2021. Process prediction with digital twins. In Proceedings of the Companion Proceedings ACM/IEEE 24th International Conference on Model Driven Engineering Languages and Systems. IEEE. DOI : https://doi.org/10.1109/MODELS-C53483.2021.00032

[23] Manfred Broy. 2006. Challenges in automotive software engineering. In Proceedings of the 28th International Conference on Software Engineering. ACM, 33-42. DOI : https://doi.org/10.1145/1134285.1134292

[24] Koen Bruynseels, Filippo Santoni de Sio, and Jeroen van den Hoven. 2018. Digital twins in health care: Ethical implications of an emerging engineering paradigm. Frontiers in Genetics 9 (2018), 31. DOI : https://doi.org/10.3389/ fgene.2018.00031

[25] Armin F. Buckhorst, Benjamin Montavon, Dominik Wolfschläger, Melanie Buchsbaum, Amir Shahidi, Henning Petruck, Ike Kunze, Jan Pennekamp, Christian Brecher, Mathias Hüsing, Burkhard Corves, Verena Nitsch, Klaus Wehrle, and Robert H. Schmitt. 2021. Holarchy for line-less mobile assembly systems operation in the context of the internet of production. Procedia CIRP 99 (2021), 448-453. DOI : https://doi.org/10.1016/j.procir.2021.03.064

[26] Bundesministerium für Bildung und Forschung. 2015. Industrie 4.0. Retrieved May 26, 2021 from https://www.bmbf. de/de/zukunftsprojekt-industrie-4-0-848.html.

[27] Arvid Butting, Robert Eikermann, Oliver Kautz, Bernhard Rumpe, and Andreas Wortmann. 2018. Modeling language variability with reusable language components. In Proceedings of the 22nd International Systems and Software Product Line Conference. ACM, 65-75. DOI : https://doi.org/10.1145/3233027.3233037

[28] Arvid Butting, Jerome Pfeiffer, Bernhard Rumpe, and Andreas Wortmann. 2020. A compositional framework for systematic modeling language reuse. In Proceedings of the 23rd ACM/IEEE International Conference on Model Driven Engineering Languages and Systems. ACM, 35-46. DOI : https://doi.org/10.1145/3365438.3410934

[29] André Calero Valdez, Philipp Brauner, Anne Kathrin Schaar, Andreas Holzinger, and Martina Ziefle. 2015. Reducing complexity with simplicity - usability methods for industry 4.0. In Proceedings of the 19th Triennial Congress of the International Ergonomics Association. IEA. DOI : https://doi.org/10.13140/RG.2.1.4253.6809

[30] Catapult. 2013. High Value Manufacturing Catapult. Retrieved May 26, 2021 from https://hvm.catapult.org.uk/.

[31] Fabricio E. Rodriguez Cesen, Levente Csikor, Carlos Recalde, Christian Esteve Rothenberg, and Gergely Pongrácz. 2020. Towards low latency industrial robot control in programmable data planes. In Proceedings of the 6th IEEE Conference on Network Softwarization. IEEE, 165-169. DOI : https://doi.org/10.1109/NetSoft48620.2020.9165531

[32] Arnab Chakrabarti, Christoph Quix, Sandra Geisler, Jaroslav Pullmann, Artur Khromov, and Matthias Jarke. 2018. Goal-oriented modelling of relations and dependencies in data marketplaces. In Proceedings of the 11th International $i^{*}$ Workshop co-located with the 30th International Conference on Advanced Information Systems Engineering, Vol. 2118.

[33] Benoit Combemale, Jörg Kienzle, Gunter Mussbacher, Olivier Barais, Erwan Bousse, Walter Cazzola, Philippe Collet, Thomas Degueule, Robert Heinrich, Jean-Marc Jézéquel, Manuel Leduc, Tanja Mayerhofer, Sébastien Mosser, Matthias Schöttle, Misha Strittmatter, and Andreas Wortmann. 2018. Concern-oriented language development : Fostering reuse in language engineering. Computer Languages, Systems \& Structures 54 (2018), 139-155. DOI: https: //doi.org/10.1016/j.cl.2018.05.004

[34] Sara J. Czaja, Walter R. Boot, Neil Charness, and Wendy A. Rogers. 2009. Designing for Older Adults: Principles and Creative Human Factors Approaches (3rd ed.). CRC Press. DOI : https://doi.org/10.1201/b22189

[35] $\mathrm{Li} \mathrm{Da} \mathrm{Xu,} \mathrm{Wu} \mathrm{He,} \mathrm{and} \mathrm{Shancang} \mathrm{Li.} \mathrm{2014.} \mathrm{Internet} \mathrm{of} \mathrm{things} \mathrm{in} \mathrm{industries:} \mathrm{A} \mathrm{survey.} \mathrm{IEEE} \mathrm{Transactions} \mathrm{on} \mathrm{Industrial}$ Informatics 10, 4 (2014), 2233-2243. DOI : https://doi.org/10.1109/TII.2014.2300753

[36] Li Da Xu, Eric L. Xu, and Ling Li. 2018. Industry 4.0: State of the art and future trends. International fournal of Production Research 56, 8 (2018), 2941-2962. DOI : https://doi.org/10.1080/00207543.2018.1444806

[37] James B. Dabney and Thomas L. Harman. 2003. Mastering Simulink (1st ed.). Pearson.

[38] Markus Dahlmanns, Johannes Lohmöller, Ina Berenice Fink, Jan Pennekamp, Klaus Wehrle, and Martin Henze. 2020. Easing the conscience with OPC UA: An internet-wide study on insecure deployments. In Proceedings of the ACM Internet Measurement Conference. ACM, 101-110. DOI : https://doi.org/10.1145/3419394.3423666

[39] Markus Dahlmanns, Johannes Lohmöller, Jan Pennekamp, Jörn Bodenhausen, Klaus Wehrle, and Martin Henze. 2022. Missed opportunities: Measuring the untapped TLS support in the industrial Internet of Things. In Proceedings of the 
17th ACM ASIA Conference on Computer and Communications Security. ACM. DOI : https://doi.org/10.1145/3488932. 3497762

[40] Markus Dahlmanns, Jan Pennekamp, Ina Berenice Fink, Bernd Schoolmann, Klaus Wehrle, and Martin Henze. 2021. Transparent end-to-end security for publish/subscribe communication in cyber-physical systems. In Proceedings of the 1st ACM Workshop on Secure and Trustworthy Cyber-Physical Systems. ACM, 78-87. DOI : https://doi.org/10.1145/ 3445969.3450423

[41] Manuela Dalibor, Nico Jansen, Judith Michael, Bernhard Rumpe, and Andreas Wortmann. 2019. Towards sustainable systems engineering-integrating tools via component and connector architectures. In Antriebstechnisches Kolloquium 2019: Tagungsband zur Konferenz. Books on Demand, 121-133.

[42] Manuela Dalibor, Nico Jansen, Bernhard Rumpe, Louis Wachtmeister, and Andreas Wortmann. 2019. Model-driven systems engineering for virtual product design. In Proceedings of the ACM/IEEE 22nd International Conference on Model Driven Engineering Languages and Systems Companion. IEEE, 430-435. DOI : https://doi.org/10.1109/MODELSC.2019.00069

[43] Manuela Dalibor, Judith Michael, Bernhard Rumpe, Simon Varga, and Andreas Wortmann. 2020. Towards a modeldriven architecture for interactive digital twin cockpits. In Proceedings of the 39th International Conference on Conceptual Modeling, Vol. 12400. Springer, 377-387. DOI : https://doi.org/10.1007/978-3-030-62522-1_28

[44] Dominik Dellermann, Philipp Ebel, Matthias Söllner, and Jan Marco Leimeister. 2019. Hybrid intelligence. Business \& Information Systems Engineering 61, 5 (2019), 637-643. DOI : https://doi.org/10.1007/s12599-019-00595-2

[45] Derek Doran, Sarah Schulz, and Tarek R. Besold. 2017. What does explainable AI really mean? A new conceptualization of perspectives. In Proceedings of the 1st International Workshop on Comprehensibility and Explanation in AI and ML, Vol. 2071.

[46] Mica R. Endsley. 2017. From here to autonomy: Lessons learned from human-automation research. Human Factors 59, 1 (2017), 5-27. DOI : https://doi.org/10.1177/0018720816681350

[47] Paola Fantini, Marta Pinzone, and Marco Taisch. 2020. Placing the operator at the centre of industry 4.0 design: Modelling and assessing human activities within cyber-physical systems. Computers \& Industrial Engineering 139 (2020), 105058. DOI : https://doi.org/10.1016/j.cie.2018.01.025

[48] Oliver Fisher, Nicholas Watson, Laura Porcu, Darren Bacon, Martin Rigley, and Rachel L. Gomes. 2018. Cloud manufacturing as a sustainable process manufacturing route. Journal of Manufacturing Systems 47 (2018), 53-68. DOI : https://doi.org/10.1016/j.jmsy.2018.03.005

[49] Robert France and Bernhard Rumpe. 2007. Model-driven development of complex software: A research roadmap. In Proceedings of the Future of Software Engineering. IEEE, 37-54. DOI : https://doi.org/10.1109/FOSE.2007.14

[50] Enzo Morosini Frazzon, Jens Hartmann, Thomas Makuschewitz, and Bernd Scholz-Reiter. 2013. Towards socio-cyberphysical systems in production networks. Procedia CIRP 7 (2013), 49-54. DOI : https://doi.org/10.1016/j.procir.2013.05. 009

[51] Sanford Friedenthal, Alan Moore, and Rick Steiner. 2014. A Practical Guide to SysML: The Systems Modeling Language (3rd ed.). Morgan Kaufmann.

[52] Günter Gans, Matthias Jarke, Stefanie Kethers, and Gerhard Lakemeyer. 2003. Continuous requirements management for organisation networks: A (dis) trust-based approach. Requirements Engineering 8, 1 (2003), 4-22. DOI : https://doi. org/10.1007/s00766-002-0163-8

[53] Jie Gao, Yinliang Yao, Valerie C. Y. Zhu, Linyan Sun, and Lin Lin. 2011. Service-oriented manufacturing: A new product pattern and manufacturing paradigm. Journal of Intelligent Manufacturing 22 (2011), 435-446. DOI : https: //doi.org/10.1007/s10845-009-0301-y

[54] Arkadii Gerasimov, Judith Michael, Lukas Netz, Bernhard Rumpe, and Simon Varga. 2020. Continuous transition from model-driven prototype to full-size real-world enterprise information systems. In Proceedings of the 25th Americas Conference on Information Systems. AIS, 1-10.

[55] René Glebke, Martin Henze, Klaus Wehrle, Philipp Niemietz, Daniel Trauth, Patrick Mattfeld, and Thomas Bergs. 2019. A case for integrated data processing in large-scale cyber-physical systems. In Proceedings of the 52nd Hawaii International Conference on System Sciences. AIS, 7252-7261. DOI : https://doi.org/10.24251/HICSS.2019.871

[56] René Glebke, Johannes Krude, Ike Kunze, Jan Rüth, Felix Senger, and Klaus Wehrle. 2019. Towards executing computer vision functionality on programmable network devices. In Proceedings of the 1st ACM CoNEXT Workshop on Emerging in-Network Computing Paradigms. ACM, 15-20. DOI : https://doi.org/10.1145/3359993.3366646

[57] Lars Gleim, Jan Pennekamp, Martin Liebenberg, Melanie Buchsbaum, Philipp Niemietz, Simon Knape, Alexander Epple, Simon Storms, Daniel Trauth, Thomas Bergs, Christian Brecher, Stefan Decker, Gerhard Lakemeyer, and Klaus Wehrle. 2020. FactDAG: Formalizing data interoperability in an internet of production. IEEE Internet of Things fournal 7, 4 (2020), 3243-3253. DOI : https://doi.org/10.1109/JIOT.2020.2966402

[58] Kate Goddard, Abdul Roudsari, and Jeremy C. Wyatt. 2012. Automation bias: A systematic review of frequency, effect mediators, and mitigators. Fournal of the American Medical Informatics Association 19, 1 (2012), 121-127. DOI : https: //doi.org/10.1136/amiajnl-2011-000089 
[59] Pavel Golodoniuc, Nicholas J. Car, and Jens Klump. 2017. Distributed persistent identifiers system design. Data Science fournal 16, 34 (2017), 34:1-34:12. DOI : https://doi.org/10.5334/dsj-2017-034

[60] David Gunning. 2019. APICS Dictionary, (16th ed.). ASCM.

[61] Rihan Hai. 2020. Data Integration and Metadata Management in Data Lakes. Ph. D. Dissertation. RWTH Aachen University. DOI : https://doi.org/10.18154/RWTH-2020-08233

[62] Robert Heim, Pedram Mir Seyed Nazari, Bernhard Rumpe, and Andreas Wortmann. 2016. Compositional language engineering using generated, extensible, static type-safe visitors. In Proceedings of the 12th European Conference on Modelling Foundations and Applications, Vol. 9764. Springer, 67-82. DOI : https://doi.org/10.1007/978-3-319-42061-5_5

[63] Martin Henze. 2020. The quest for secure and privacy-preserving cloud-based industrial cooperation. In Proceedings of the Conference on Communications and Network Security. IEEE. DOI : https://doi.org/10.1109/CNS48642.2020. 9162199

[64] Jens Hiller, Jan Pennekamp, Markus Dahlmanns, Martin Henze, Andriy Panchenko, and Klaus Wehrle. 2019. Tailoring onion routing to the internet of things: Security and privacy in untrusted environments. In Proceedings of the 27th International Conference on Network Protocols. IEEE. DOI : https://doi.org/10.1109/ICNP.2019.8888033

[65] Kevin Anthony Hoff and Masooda Bashir. 2015. Trust in automation: Integrating empirical evidence on factors that influence trust. Human Factors 57, 3 (2015), 407-434. DOI : https://doi.org/10.1177/0018720814547570

[66] Till Hofmann, Nicolas Limpert, Victor Mataré, Alexander Ferrein, and Gerhard Lakemeyer. 2019. Winning the robocup logistics league with fast navigation, precise manipulation, and robust goal reasoning. In Proceedings of the RoboCup 2019: Robot World Cup XXIII, Vol. 11531. Springer, 504-516. DOI : https://doi.org/10.1007/978-3-030-356996_41

[67] Till Hofmann, Nicolas Limpert, Victor Mataré, Sebastian Schönitz, Tim Niemueller, Alexander Ferrein, and Gerhard Lakemeyer. 2018. The Carologistics RoboCup Logistics Team 2018. Technical Report. RWTH Aachen University and Aachen University of Applied Sciences.

[68] Katrin Hölldobler, Bernhard Rumpe, and Andreas Wortmann. 2018. Software language engineering in the large: Towards composing and deriving languages. Computer Languages, Systems \& Structures 54 (2018), 386-405. DOI : https: //doi.org/10.1016/j.cl.2018.08.002

[69] Gregor Höpfner, Georg Jacobs, Thilo Zerwas, Imke Drave, Joerg Berroth, Christian Guist, Bernhard Rumpe, and Jens Kohl. 2021. Model-based design workflows for cyber-physical systems applied to an electric-mechanical coolant pump. In Proceedings of the 19th Drive Train Technology Conference, Vol. 1097. IOP Publishing. DOI: https://doi.org/ 10.1088/1757-899X/1097/1/012004

[70] Wallace J. Hopp and Mark L. Spearman. 2004. To pull or not to pull: What is the question? Manufacturing \& Service Operations Management 6, 2 (2004), 133-148. DOI : https://doi.org/10.1287/msom.1030.0028

[71] Industrial Value Chain Initiative. 2019. What is IVI? - Industrial Value Chain Initiative. Retrieved May 26, 2021 from https://iv-i.org/wp/en/about-us/whatsivi/.

[72] International Trade Administration. 2021. Korea - Manufacturing Technology - Smart Factory. Retrieved May 26, 2021 from https://www.privacyshield.gov/article?id=Korea-Manufacturing-Technology-Smart-Factory.

[73] Matthias Jarke. 2020. Data sovereignty and the internet of production. In Proceedings of the 32nd International Conference on Advanced Information Systems Engineering, Vol. 12127. Springer, 549-558. DOI : https://doi.org/10.1007/9783-030-49435-3_34

[74] Matthias Jarke and Christoph Quix. 2017. On Warehouses, Lakes, and Spaces: The Changing Role of Conceptual Modeling for Data Integration. Springer, 231-245. DOI : https://doi.org/10.1007/978-3-319-67271-7_16

[75] Szilárd Jaskó, Adrienn Skrop, Tibor Holczinger, Tibor Chován, and János Abonyi. 2020. Development of manufacturing execution systems in accordance with industry 4.0 requirements: A review of standard- and ontologybased methodologies and tools. Computers in Industry 123 (2020), 103300. DOI : https://doi.org/10.1016/j.compind. 2020.103300

[76] Manfred A. Jeusfeld, Matthias Jarke, and John Mylopoulos. 2009. Metamodeling for Method Engineering. The MIT Press.

[77] David Jones, Chris Snider, Aydin Nassehi, Jason Yon, and Ben Hicks. 2020. Characterising the digital twin: A systematic literature review. CIRP fournal of Manufacturing Science and Technology 29, Part A (2020), 36-52. DOI : https: //doi.org/10.1016/j.cirpj.2020.02.002

[78] Bzhwen A. Kadir, Ole Broberg, and Carolina Souza da Conceicao. 2019. Current research and future perspectives on human factors and ergonomics in industry 4.0. Computers \& Industrial Engineering 137 (2019), 106004. DOI: https: //doi.org/10.1016/j.cie.2019.106004

[79] Ravi Kalaiarasan, Jan Olhager, Magnus Wiktorsson, and Yongkuk Jeong. 2020. Production logistics visibility - perspectives, principles and prospects. In Proceedings of the 9th Swedish Production Symposium on Advances in Transdisciplinary Engineering, Vol. 13. IOS Press, 501-510. DOI : https://doi.org/10.3233/ATDE200188 
[80] Nasr Kasrin, Maliha Qureshi, Simon Steuer, and Daniela Nicklas. 2018. Semantic data management for experimental manufacturing technologies. Datenbank-Spektrum 18, 1 (2018), 27-37. DOI:https://doi.org/10.1007/ s13222-018-0274-0

[81] Jörg Christian Kirchhof, Judith Michael, Bernhard Rumpe, Simon Varga, and Andreas Wortmann. 2020. Model-driven digital twin construction: Synthesizing the integration of cyber-physical systems with their information systems. In Proceedings of the 23rd ACM/IEEE International Conference on Model Driven Engineering Languages and Systems. ACM, 90-101. DOI : https://doi.org/10.1145/3365438.3410941

[82] István Koren. 2021. A standalone webassembly development environment for the internet of things. In Proceedings of the 21st International Conference on Web Engineering, Vol. 12706. Springer, 353-360. DOI : https://doi.org/10.1007/9783-030-74296-6_27

[83] István Koren, Stefan Braun, Marc Van Dyck, and Matthias Jarke. 2021. Dynamic strategic modeling for alliancedriven data platforms: The case of smart farming. In Proceedings of the 33rd International Conference on Advanced Information Systems Engineering, Vol. 424. Springer, 92-99. DOI : https://doi.org/10.1007/978-3-030-79108-7_11

[84] Dejan Kovachev, Yiwei Cao, Ralf Klamma, and Matthias Jarke. 2011. Learn-as-you-go: New ways of cloud-based micro-learning for the mobile web. In Proceedings of the 10th International Conference on Advances in Web-Based Learning, Vol. 7048. Springer, 51-61. DOI : https://doi.org/10.1007/978-3-642-25813-8_6

[85] Sathish A. P. Kumar, R. Madhumathi, Pethuru Raj Chelliah, Lei Tao, and Shangguan Wang. 2018. A novel digital twin-centric approach for driver intention prediction and traffic congestion avoidance. fournal of Reliable Intelligent Environments 4, 4 (2018), 199-209. DOI : https://doi.org/10.1007/s40860-018-0069-y

[86] Ike Kunze, René Glebke, Jan Scheiper, Matthias Bodenbenner, Robert H. Schmitt, and Klaus Wehrle. 2021. Investigating the applicability of in-network computing to industrial scenarios. In Proceedings of the 4th IEEE International Conference on Industrial Cyber-Physical Systems. IEEE, 334-340. DOI : https://doi.org/10.1109/ICPS49255.2021.9468247

[87] Ike Kunze, Philipp Niemietz, Liam Tirpitz, René Glebke, Daniel Trauth, Thomas Bergs, and Klaus Wehrle. 2021. Detecting out-of-control sensor signals in sheet metal forming using in-network computing. In Proceedings of the 30th International Symposium on Industrial Electronics. IEEE.

[88] Asma Ladj, Zhiqiang Wang, Oussama Meski, Farouk Belkadi, Mathieu Ritou, and Catherine Da Cunha. 2021. A knowledge-based digital shadow for machining industry in a digital twin perspective. fournal of Manufacturing Systems 58, Part B (2021), 168-179. DOI : https://doi.org/10.1016/j.jmsy.2020.07.018

[89] Brenden M. Lake, Tomer D. Ullman, Joshua B. Tenenbaum, and Samuel J. Gershman. 2017. Building machines that learn and think like people. Behavioral and Brain Sciences 40, 2017 (2017), 253:1-253:72. DOI : https://doi.org/10.1017/ S0140525X16001837

[90] Paulo Leitão and Stamatis Karnouskos. 2015. Industrial Agents: Emerging Applications of Software Agents in Industry (1st ed.). Morgan Kaufmann.

[91] Paulo Leitao, Stamatis Karnouskos, Luis Ribeiro, Jay Lee, Thomas Strasser, and Armando W. Colombo. 2016. Smart agents in industrial cyber-physical systems. Proceedings of the IEEE 104, 5 (2016), 1086-1101. DOI : https://doi.org/10. 1109/JPROC.2016.2521931

[92] Martin Liebenberg and Matthias Jarke. 2020. Information systems engineering with digital shadows: Concept and case studies. In Proceedings of the 32nd International Conference on Advanced Information Systems Engineering, Vol. 12127. Springer, 70-84. DOI : https://doi.org/10.1007/978-3-030-49435-3_5

[93] Luca Liehner, Philipp Brauner, Anne Kathrin Schaar, and Martina Ziefle. 2021. Delegation of moral tasks to automated agents - The impact of risk and context on trusting a machine to perform a task. IEEE Transactions on Technology and Society (2021). DOI : https://doi.org/10.1109/TTS.2021.3118355

[94] Kendrik Yan Hong Lim, Pai Zheng, and Chun-Hsien Chen. 2020. A state-of-the-art survey of digital twin: Techniques, engineering product lifecycle management and business innovation perspectives. fournal of Intelligent Manufacturing 31, 6 (2020), 1313-1337. DOI : https://doi.org/10.1007/s10845-019-01512-w

[95] Fábio Lima, Caroline Nogueira de Carvalho, Mayara B. S. Acardi, Eldiane Gomes dos Santos, Gabriel Bastos de Miranda, Rodrigo Filev Maia, and Alexandre Augusto Massote. 2019. Digital manufacturing tools in the simulation of collaborative robots: Towards industry 4.0. Brazilian fournal of Operations \& Production Management 16, 2 (2019), 261-280. DOI : https://doi.org/10.14488/BJOPM.2019.v16.n2.a8

[96] Johannes Lipp, Maximilian Rudack, Uwe Vroomen, and Andreas Bührig-Polaczek. 2020. When to collect what? Optimizing data load via process-driven data collection. In Proceedings of the 22nd International Conference on Enterprise Information Systems. SCITEPRESS, 220-225. DOI : https://doi.org/10.5220/0009439502200225

[97] Yongkui Liu and Xun Xu. 2017. Industry 4.0 and cloud manufacturing: A comparative analysis. fournal of Manufacturing Science and Engineering 139, 3 (2017), 034701:1-034701:8. DOI : https://doi.org/10.1115/1.4034667

[98] Arndt Lüder and Nicole Schmidt. 2017. AutomationML in a Nutshell. Springer, 213-258. DOI : https://doi.org/10.1007/ 978-3-662-53248-5_61 
[99] Deborah L. McGuinness and Frank van Harmelen. 2004. OWL Web Ontology Language Overview. W3C Recommendation.

[100] Sheila A. McIlraith and Tran Cao Son. 2002. Adapting golog for composition of semantic web services. In Proceedings of the 8th International Conference on Principles of Knowledge Representation and Reasoning. Morgan Kaufmann, 482-496.

[101] MERICS. 2016. Made in China 2025. Retrieved May 26, 2021 from https://merics.org/en/report/made-china- 2025.

[102] Alexander Mertens, Sebastian Pütz, Philipp Brauner, Florian Brillowski, Nadine Buczak, Hannah Dammers, Marc van Dyck, Iris Kong, Peter Königs, Frauke Kordtomeikel, Niklas Rodemann, Anne Kathrin Schaar, Linda SteuerDankert, Shari Wlecke, Thomas Gries, Carmen Leicht-Scholten, Saskia K. Nagel, Frank T. Piller, Günther Schuh, Martina Ziefle, and Verena Nitsch. 2021. Human digital shadow: Data-based modeling of users and usage in the internet of production. In Proceedings of the 2021 14th International Conference on Human System Interaction. IEEE. DOI : https://doi.org/10.1109/HSI52170.2021.9538729

[103] Richard Meyes, Hasan Tercan, Thomas Thiele, Alexander Krämer, Julian Heinisch, Martin Liebenberg, Gerhard Hirt, Christian Hopmann, Gerhard Lakemeyer, Tobias Meisen, and Sabina Jeschke. 2018. Interdisciplinary data driven production process analysis for the internet of production. Procedia Manufacturing 26 (2018), 1065-1076. DOI : https: //doi.org/10.1016/j.promfg.2018.07.143

[104] Judith Michael, Lukas Netz, Bernhard Rumpe, and Simon Varga. 2019. Towards privacy-preserving IoT systems using model driven engineering. In Proceedings of the Workshop on Model-Driven Engineering for the Internet of Things, Vol. 2442. CEUR Workshop Proceedings, 15-22.

[105] Judith Michael, Bernhard Rumpe, and Simon Varga. 2020. Human behavior, goals and model-driven software engineering for assistive systems. In Proceedings of the 10th International Workshop on Enterprise Modeling and Information Systems Architectures, Vol. 2628. CEUR Workshop Proceedings, 11-18.

[106] Roberto Minerva, Gyu Myoung Lee, and Noel Crespi. 2020. Digital twin in the IoT context: A survey on technical features, scenarios, and architectural models. Proceedings of the IEEE 108, 10 (2020), 1785-1824. DOI : https://doi.org/ 10.1109/JPROC.2020.2998530

[107] Dietmar P. F. Möller. 2016. Digital Manufacturing/Industry 4.0. Springer, 307-375. DOI : https://doi.org/10.1007/978-3319-25178-3_7

[108] Michael F. Molnar. 2017. The U.S. Advanced Manufacturing Initiative. Technical Report. NIST. Retrieved from https: //www.nist.gov/system/files/documents/2017/04/28/Molnar_091211.pdf.

[109] Benoit Montreuil. 2011. Toward a physical internet: Meeting the global logistics sustainability grand challenge. Logistics Research 3, 2 (2011), 71-87. DOI : https://doi.org/10.1007/s12159-011-0045-x

[110] Julian M. Müller, Johannes W. Veile, and Kai-Ingo Voigt. 2020. Prerequisites and incentives for digital information sharing in industry 4.0-an international comparison across data types. Computers \& Industrial Engineering 148 (2020), 106733. DOI : https://doi.org/10.1016/j.cie.2020.106733

[111] Boris Otto, Sören Auer, Jan Cirullies, Jan Jürjens, Nadja Menz, Jochen Schon, and Sven Wenzel. 2016. Industrial Data Space: Digital Souvereignity over Data. White Paper. Fraunhofer. DOI : https://doi.org/10.13140/RG.2.1.2673.0649

[112] Boris Otto and Matthias Jarke. 2019. Designing a multi-sided data platform: Findings from the international data spaces case. Electronic Markets 29, 4 (2019), 561-580. DOI : https://doi.org/10.1007/s12525-019-00362-x

[113] Cristina Paniagua and Jerker Delsing. 2020. Industrial frameworks for internet of things: A survey. IEEE Systems fournal 15, 1 (2020), 1149-1159. DOI : https://doi.org/10.1109/JSYST.2020.2993323

[114] Raja Parasuraman and Victor Riley. 1997. Humans and automation: Use, misuse, disuse, abuse. Human Factors 39, 2 (1997), 230-253. DOI : https://doi.org/10.1518/001872097778543886

[115] Tobias Pauli, Erwin Fielt, and Martin Matzner. 2021. Digital industrial platforms. Business \& Information Systems Engineering 63, 2 (2021), 181-190. DOI : https://doi.org/10.1007/s12599-020-00681-w

[116] Pieter Pauwels and Walter Terkaj. 2016. EXPRESS to OWL for construction industry: Towards a recommendable and usable ifcOWL ontology. Automation in Construction 63 (2016), 100-133. DOI : https://doi.org/10.1016/j.autcon.2015. 12.003

[117] Jan Pennekamp, Fritz Alder, Roman Matzutt, Jan Tobias Mühlberg, Frank Piessens, and Klaus Wehrle. 2020. Secure end-to-end sensing in supply chains. In Proceedings of the Conference on Communications and Network Security. IEEE. DOI : https://doi.org/10.1109/CNS48642.2020.9162337

[118] Jan Pennekamp, Lennart Bader, Roman Matzutt, Philipp Niemietz, Daniel Trauth, Martin Henze, Thomas Bergs, and Klaus Wehrle. 2020. Private multi-hop accountability for supply chains. In Proceedings of the International Conference on Communications Workshops. IEEE. DOI : https://doi.org/10.1109/ICCWorkshops49005.2020.9145100

[119] Jan Pennekamp, Erik Buchholz, Yannik Lockner, Markus Dahlmanns, Tiandong Xi, Marcel Fey, Christian Brecher, Christian Hopmann, and Klaus Wehrle. 2020. Privacy-preserving production process parameter exchange. In Proceedings of the 36th Annual Computer Security Applications Conference. ACM, 510-525. DOI : https://doi.org/10.1145/ 3427228.3427248 
[120] Jan Pennekamp, Markus Dahlmanns, Lars Gleim, Stefan Decker, and Klaus Wehrle. 2019. Security considerations for collaborations in an industrial IoT-based lab of labs. In Proceedings of the 3rd IEEE Global Conference on Internet of Things. IEEE. DOI : https://doi.org/10.1109/GCIoT47977.2019.9058413

[121] Jan Pennekamp, René Glebke, Martin Henze, Tobias Meisen, Christoph Quix, Rihan Hai, Lars Gleim, Philipp Niemietz, Maximilian Rudack, Simon Knape, Alexander Epple, Daniel Trauth, Uwe Vroomen, Thomas Bergs, Christian Brecher, Andreas Bührig-Polaczek, Matthias Jarke, and Klaus Wehrle. 2019. Towards an infrastructure enabling the internet of production. In Proceedings of the International Conference on Industrial Cyber Physical Systems. IEEE, 31-37. DOI : https: //doi.org/10.1109/ICPHYS.2019.8780276

[122] Jan Pennekamp, Martin Henze, Simo Schmidt, Philipp Niemietz, Marcel Fey, Daniel Trauth, Thomas Bergs, Christian Brecher, and Klaus Wehrle. 2019. Dataflow challenges in an Internet of Production: A Security \& Privacy Perspective. In Proceedings of the ACM Workshop on Cyber-Physical Systems Security \& Privacy. ACM, 27-38. DOI : https://doi.org/ $10.1145 / 3338499.3357357$

[123] Jan Pennekamp, Roman Matzutt, Salil S. Kanhere, Jens Hiller, and Klaus Wehrle. 2021. The road to accountable and dependable manufacturing. Automation 2, 3 (2021), 202-219. DOI : https://doi.org/10.3390/automation2030013

[124] Jan Pennekamp, Patrick Sapel, Ina Berenice Fink, Simon Wagner, Sebastian Reuter, Christian Hopmann, Klaus Wehrle, and Martin Henze. 2020. Revisiting the privacy needs of real-world applicable company benchmarking. In Proceedings of the 8th Workshop on Encrypted Computing \& Applied Homomorphic Cryptography. HomomorphicEncryption.org, 31-44. DOI : https://doi.org/10.25835/0072999

[125] Ralf Philipsen, Philipp Brauner, Sebastian Stiller, Simone Runge, Robert Schmitt, and Martina Ziefle. 2014. Understanding and Supporting Decision Makers in Quality Management of Production Networks. CRC Press, 94-105.

[126] Michael Polanyi. 1966. The Tacit Dimension. University of Chicago Press.

[127] Jinghai Rao and Xiaomeng Su. 2004. A survey of automated web service composition methods. In Proceedings of the 1st International Workshop on Semantic Web Services and Web Process Composition, Vol. 3387. Springer, 43-54. DOI : https://doi.org/10.1007/978-3-540-30581-1_5

[128] Michael Riesener, Günther Schuh, Christian Dölle, and Christian Tönnes. 2019. The digital shadow as enabler for data analytics in product life cycle management. Procedia CIRP 80 (2019), 729-734.

[129] Bernhard Rumpe. 2017. Agile Modeling with UML: Code Generation, Testing, Refactoring (1st ed.). Springer.

[130] Bernhard Rumpe, Katrin Hölldobler, and Oliver Kautz. 2021. MontiCore Language Workbench and Library Handbook: Edition 2021. Shaker.

[131] Stuart J. Russell and Peter Norvig. 2009. Artificial Intelligence: A Modern Approach (3rd ed.). Pearson.

[132] Jan Rüth, René Glebke, Klaus Wehrle, Vedad Causevic, and Sandra Hirche. 2018. Towards in-network industrial feedback control. In Proceedings of the Morning Workshop on In-Network Computing. ACM, 14-19. DOI : https://doi. org $/ 10.1145 / 3229591.3229592$

[133] Filippo Santoni de Sio and Giulio Mecacci. 2021. Four responsibility gaps with artificial intelligence: Why they matter and how to address them. Philosophy \& Technology 34, 4 (2021), 1057-1084. https://doi.org/10.1007/s13347021-00450-x

[134] Frank Schnicke, Thomas Kuhn, and Pablo Oliveira Antonino. 2020. Enabling industry 4.0 service-oriented architecture through digital twins. In Proceedings of the 14th European Conference on Software Architecture, Vol. 1269. Springer, 490-503. DOI : https://doi.org/10.1007/978-3-030-59155-7_35

[135] Günther Schuh, Constantin Häfner, Christian Hopmann, Bernhard Rumpe, Matthias Brockmann, Andreas Wortmann, Judith Maibaum, Manuela Dalibor, Pascal Bibow, Patrick Sapel, and Moritz Kröger. 2020. Effizientere produktion mit digitalen schatten. ZWF Zeitschrift für wirtschaftlichen Fabrikbetrieb 115, Special (2020), 105-107. DOI : https: //doi.org/10.3139/104.112339

[136] Sculpteo. 2017. Digital Manufacturing - The Factory of the Future is Here Today. Retrieved August 31, 2021 from https://www.industryweek.com/technology-and-iiot/article/21995642/digital-manufacturing-the-factory-ofthe-future-is-here-today.

[137] Ajit Sharma, Philip Zanotti, and Laxmi P. Musunur. 2019. Enabling the electric future of mobility: Robotic automation for electric vehicle battery assembly. IEEE Access 7 (2019), 170961-170991. DOI : https://doi.org/10.1109/ACCESS.2019. 2953712

[138] Vladimir A. Shekhovtsov, Suneth Ranasinghe, Heinrich C. Mayr, and Judith Michael. 2018. Domain specific models as system links. In Proceedings of the Advances in Conceptual Modeling Workshops, Vol. 11158. Springer, 330-340. DOI : https://doi.org/10.1007/978-3-030-01391-2_37

[139] Julia Siderska and Khair S. Jadaan. 2018. Cloud manufacturing: A service-oriented manufacturing paradigm. A review paper. Engineering Management in Production and Services 10, 1 (2018), 22-31. DOI : https://doi.org/10.1515/emj-20180002 
[140] Emiliano Sisinni, Abusayeed Saifullah, Song Han, Ulf Jennehag, and Mikael Gidlund. 2018. Industrial internet of things: Challenges, opportunities, and directions. IEEE Transactions on Industrial Informatics 14, 11 (2018), 4724-4734. DOI : https://doi.org/10.1109/TII.2018.2852491

[141] Shirin Sohrabi, Nataliya Prokoshyna, and Sheila A. McIlraith. 2009. Web service composition via the customization of golog programs with user preferences. In Proceedings of the Conceptual Modeling: Foundations and Applications, Vol. 5600. Springer, 319-334. DOI : https://doi.org/10.1007/978-3-642-02463-4_17

[142] Tim Stock and Günther Seliger. 2016. Opportunities of sustainable manufacturing in industry 4.0. Procedia CIRP 40 (2016), 536-541. DOI : https://doi.org/10.1016/j.procir.2016.01.129

[143] Fei Tao, He Zhang, Ang Liu, and Andrew Y. C. Nee. 2018. Digital twin in industry: State-of-the-art. IEEE Transactions on Industrial Informatics 15, 4 (2018), 2405-2415. DOI : https://doi.org/10.1109/TII.2018.2873186

[144] Syed A. M. Tofail, Elias P. Koumoulos, Amit Bandyopadhyay, Susmita Bose, Lisa O’Donoghue, and Costas Charitidis. 2018. Additive manufacturing: Scientific and technological challenges, market uptake and opportunities. Materials Today 21, 1 (2018), 22-37. DOI : https://doi.org/10.1016/j.mattod.2017.07.001

[145] Wil M. P. van der Aalst. 2016. Process Mining: Data Science in Action (2nd ed.). Springer. DOI : https://doi.org/10.1007/ 978-3-662-49851-4

[146] Wil M. P. van der Aalst, Shengnan Guo, and Pierre Gorissen. 2015. Comparative process mining in education: An approach based on process cubes. In Proceedings of the 3rd International Symposium on Data-Driven Process Discovery and Analysis, Vol. 203. Springer, 110-134. DOI : https://doi.org/10.1007/978-3-662-46436-6_6

[147] Birgit Vogel-Heuser, Markus Böhm, Felix Brodeck, Katharina Kugler, Sabine Maasen, Dorothea Pantförder, Minjie Zou, Johan Buchholz, Harald Bauer, Felix Brandl, and Udo Lindemann. 2020. Interdisciplinary engineering of cyberphysical production systems: Highlighting the benefits of a combined interdisciplinary modelling approach on the basis of an industrial case. Design Science 6 (2020), 5:1-5:36. DOI : https://doi.org/10.1017/dsj.2020.2

[148] Markus Völter, Thomas Stahl, Jorn Bettin, Arno Haase, Simon Helsen, and Krzysztof Czarnecki. 2013. Model-Driven Software Development: Technology, Engineering, Management. Wiley.

[149] Jiafu Wan, Shenglong Tang, Di Li, Muhammad Imran, Chunhua Zhang, Chengliang Liu, and Zhibo Pang. 2019. Reconfigurable smart factory for drug packing in healthcare industry 4.0. IEEE Transactions on Industrial Informatics 15, 1 (2019), 507-516. DOI : https://doi.org/10.1109/TII.2018.2843811

[150] Chris Wickens, Justin G. Hollands, Simon Banbury, and Raja Parasuraman. 2013. Engineering Psychology and Human Performance (4th ed.). Prentice Hall. DOI : https://doi.org/10.1146/annurev.ps.27.020176.001513

[151] World Economic Forum and McKinsey \& Company. 2021. Global Lighthouse Network: Reimagining Operations for Growth. White Paper. World Economic Forum.

[152] Andreas Wortmann, Olivier Barais, Benoit Combemale, and Manuel Wimmer. 2020. Modeling languages in industry 4.0: An extended systematic mapping study. Software and Systems Modeling 19, 1 (2020), 67-94. DOI : https://doi.org/ 10.1007/s10270-019-00757-6

[153] Xun Xu. 2012. From cloud computing to cloud manufacturing. Robotics and Computer-Integrated Manufacturing 28, 1 (2012), 75-86. DOI : https://doi.org/10.1016/j.rcim.2011.07.002

[154] Lu Zhen. 2012. An analytical study on service-oriented manufacturing strategies. International fournal of Production Economics 139, 1 (2012), 220-228. DOI : https://doi.org/10.1016/j.ijpe.2012.04.010

[155] Ting Zheng, Marco Ardolino, Andrea Bacchetti, and Marco Perona. 2021. The applications of industry 4.0 technologies in manufacturing context: A systematic literature review. International fournal of Production Research 59, 6 (2021), 1922-1954. DOI : https://doi.org/10.1080/00207543.2020.1824085

Received July 2020; revised October 2021; accepted November 2021 